\title{
High-resolution radio study of SNR IC 443 at low radio frequencies
}

\author{
G. Castelletti ${ }^{1,2, \star}$, G. Dubner ${ }^{1, \star}$, T. Clarke ${ }^{3}$, and N. E. Kassim ${ }^{3}$ \\ ${ }^{1}$ Instituto de Astronomía y Física del Espacio (IAFE, CONICET-UBA), CC67, Suc.28, 1428 Buenos Aires, Argentina \\ e-mail: gcastell@iafe.uba.ar \\ 2 Facultad de Ciencias Exactas y Naturales (Universidad de Buenos Aires), Argentina \\ ${ }^{3}$ Remote Sensing Division, Code 7213, Naval Research Laboratory, 4555 Overlook Avenue, SW, Washington DC, USA
}

Received 5 November 2010 / Accepted 1 April 2011

\section{ABSTRACT}

\begin{abstract}
Aims. We investigate the morphology at low radio frequencies of the supernova remnant (SNR) IC 443 in detail and accurately establish its radio continuum spectral properties.

Methods. We used the VLA in multiple configurations to produce high-resolution radio images of IC 443 at 74 and $330 \mathrm{MHz}$. From these data we produced the first sensitive, spatially resolved spectral analysis of the radio emission at long wavelengths. The changes with position in the radio spectral index were correlated with data in near infrared (NIR) from 2MASS, in gamma-rays from VERITAS, and with the molecular ${ }^{12} \mathrm{CO}(J=1-0)$ line emission.

Results. The new image at $74 \mathrm{MHz}$ has $H P B W=35^{\prime \prime}$ and $\mathrm{rms}=30 \mathrm{mJy}^{-1}$ beam ${ }^{-1}$ and at $330 \mathrm{MHz} H P B W=17^{\prime \prime}$ and rms $=$ $1.7 \mathrm{mJy}$ beam $^{-1}$. The integrated flux densities for the whole SNR are $S_{74 \mathrm{MHz}}^{\mathrm{SNR}}=470 \pm 51 \mathrm{Jy}$ and $S_{330 \mathrm{MHz}}^{\mathrm{SNR}}=248 \pm 15 \mathrm{Jy}$. Improved estimates of the integrated spectrum were derived taking a turnover into account to fit the lowest frequency measurements in the literature. Combining our measurements with existing data, we derive an integrated spectral index $\alpha_{10 \mathrm{MHz}}^{10700 \mathrm{MHz}}=-0.39 \pm 0.01$ with a free-free continuum optical depth at $330 \mathrm{MHz} \tau_{330} \sim 7 \times 10^{-4}\left(\tau_{10}=1.07\right)$; all measurements above $\sim 10 \mathrm{MHz}$ are equally consistent with a power law spectrum. For the pulsar wind nebula associated with the compact source CXOU J061705.3+222127, we calculated $S_{330 \mathrm{MHz}}^{\mathrm{PWN}}=0.23 \pm 0.05 \mathrm{Jy}, S_{1420 \mathrm{MHz}}^{\mathrm{PWN}}=0.20 \pm 0.04 \mathrm{Jy}$, and $\alpha_{330 \mathrm{MHz}}^{8460 \mathrm{MHz}} \sim 0.0$. Substantial variations are observed in spectral index between 74 and $330 \mathrm{MHz}$ across IC 443 . The flattest spectral components $(-0.25 \leq \alpha \leq-0.05)$ coincide with the brightest parts of the SNR along the eastern border, with an impressive agreement with ionic lines as observed in the 2MASS $J$ and $H$ bands. The diffuse interior of IC 443 has a spectrum steeper than found anywhere in the SNR $(-0.85 \leq \alpha \leq-0.6)$, while the southern ridge again has a flatter spectrum $(\alpha \sim-0.4)$. With the available statistics the VERITAS $\gamma$-ray emission strikingly matches the CO distribution, but no clear evidence is found for a morphological correlation between the $\mathrm{TeV}$ distribution and radio emission.

Conclusions. The excellent correspondence between the eastern radio flattest spectrum region and NIR ionic lines strongly suggests that the passage of a fast, dissociating J-type shock across the interacting molecular cloud dissociated the molecules and ionized the gas. We therefore conclude that thermal absorption at $74 \mathrm{MHz}$ ( $\tau_{74}$ up to $\sim 0.3$ ) is responsible for the localized spectral index flattening observed along the eastern border of IC 443. Towards the interior of IC 443, the spectrum is consistent with those expected from linear diffusive shock acceleration, while the flatter spectrum in the southern ridge is a consequence of the strong shock/molecular cloud interaction.
\end{abstract}

Key words. ISM: individual objects: IC 443 - ISM: supernova remnants - radio continuum: ISM - infrared: ISM gamma rays: ISM - ISM: clouds

\section{Introduction}

The importance of radio continuum studies of SNRs for understanding shock acceleration processes (Reynolds \& Ellison 1992; Anderson \& Rudnick 1996), as well as intrinsic and extrinsic interactions with ionized gas and the interstellar medium (ISM) (Dulk \& Slee 1975; Kassim 1989), has been long appreciated. However, the magnitude of the observable effects are often subtle, requiring a large leverage arm in frequency space to discern. The lack, until recently, of sufficient angular resolution and sensitivity at the lowest frequencies has slowed progress in such studies. The advent of high-resolution, low-frequency observations with instruments like the VLA and GMRT are now changing this picture. In particular, VLA subarcminute resolution imaging below $100 \mathrm{MHz}$ has been important for discerning spatially resolved intrinsic and extrinsic

\footnotetext{
* Member of the Carrera del Investigador Científico of CONICET, Argentina.
}

thermal absorption. Examples now include unshocked ejecta inside Cas A (Kassim et al. 1995), thermal filaments within the Crab nebula (Bietenholz et al. 1997), and ionized gas in the ISM along the line of sight towards W49B (Lacey et al. 2001). Later on, Brogan et al. (2005) spatially resolved the ionized boundary marking the SNR/molecular cloud (MC) interface in 3C 391, suggesting such signatures could be both common and important for delineating elusive SNR/MC interactions. Most recently, Castelletti et al. (2007) have detected strong $74 \mathrm{MHz}$ free-free absorption at the interface between the SNR W44 and the photo dissociation region of a neighboring HII region.

In this paper we extend such high-resolution, low-frequency radio studies to the classic SNR IC 443, one of the clearest previously known cases of a remnant interacting with its cloudy surroundings. We present the first, high-resolution radio studies of this object, and interpret our results in the context of recent IR and high-energy observations. IC 443 is a particularly attractive SNR for low-frequency studies both because of its large angular 
size and because its outer Galaxy location leaves it relatively well isolated from the confusing effects normally contaminating studies towards inner Galactic SNR/MC complexes.

Observed in the radio domain IC $443(\mathrm{G} 189.1+3.0)$ is among the larger SNRs in angular size cataloged in our Galaxy. It consists of two connected, roughly spherical, shells of radio synchrotron emission, which are centered at different locations (the names "shell A" and "shell B" are sometimes used to refer their locations towards the east and west halves of the remnant, Braun $\&$ Strom 1986b). On its eastern side, IC 443 has a rim-brightened morphology, while towards the western half the surface brightness is dimmer. A third incomplete and faint shell (called by Braun \& Strom 1986b, "shell C") is also evident extending beyond the northeast periphery of the remnant when the radio images are displayed with very high contrast. Based on both its morphology and soft X-ray spectrum, "shell C" was proposed to be a different SNR called G189.6+3.3, that overlaps with IC 443 (Asaoka \& Aschenbach 1994). At present it is still questionable if it is an SNR at all, and, if it is, then it is unclear whether G189.6+3.3 is interacting with IC 443 (Asaoka \& Aschenbach 1994; Leahy 2004; Lee et al. 2008).

At optical wavelengths the appearance of IC 443 resembles that observed in the radio band. Spectroscopic and photometric observations of this remnant reveal a complex filamentary structure varying in brightness and shape, which is mainly composed of Balmer-line emission, strong [CaII], [SII], [NII], [OIII], as well as faint [FeII] and [FeIII] emission regions (Fesen $\&$ Kirshner 1980). The emission-line ratios are consistent with those of a well-evolved SNR (Fesen \& Kirshner 1980).

Kinematical considerations based on optical systemic velocities place the SNR between 0.7 and $1.5 \mathrm{kpc}$ (Lozinskaya 1981), although under the assumption of a physical relationship of IC 443 with the nearby HII region Sharpless 249, it might be located slightly more distant between 1.5 and $2 \mathrm{kpc}$ (Fesen 1984). A distance of $1.5 \mathrm{kpc}$ is adopted in most of the works related to IC 443.

Different X-ray observations carried out with the Einstein Observatory (Petre et al. 1988), Ginga (Wang et al. 1992), ROSAT (Asaoka \& Aschenbach 1994), ASCA (Kawasaki et al. 2002), and XMM-Newton (Troja et al. 2006) show a bulk of thermal centrally peaked X-ray emission within the radio rim. On the basis of the radio/X-ray morphology and the X-ray properties, several authors proposed that IC 443 belongs to the class of "mixed-morphology" or "thermal-composite" SNRs (Rho \& Petre 1998).

The compact X-ray source CXOU J061705.3+222127 is thought to be the remnant of the explosion that gave rise to IC 443. This compact relic, discovered using data at high radio frequencies and in X-rays, is embedded within the remnant and is located close to its southern edge (Olbert et al. 2001). Albeit no pulsations were detected from the neutron star, the measure of its transverse velocity provides an estimate for the age of both the SNR and its associated neutron star, of about $3 \times 10^{4} \mathrm{yr}$ (Olbert et al. 2001). An alternative estimate of $\sim 3000 \mathrm{yr}$ was previously proposed by Petre et al. (1988) based on a model of its X-ray emission.

IC 443 is a prototypical case of an SNR impacting dense interstellar molecular gas. The giant molecular cloud, as mapped by $\mathrm{CO}$ and $\mathrm{HCO}^{+}$observations, forms a ring in the foreground and appears to be interacting with the remnant at several locations from north to south (Dickman et al. 1992). This phenomenon, which seems to be responsible for the multiwavelength observational picture of the remnant, was unambiguously confirmed by the presence of several $\mathrm{OH}(1720 \mathrm{MHz})$ masers as well as IR cooling lines from $\mathrm{H}_{2}$ (Rho et al. 2001; Hewitt et al. 2009, and references therein) Additionally, HI observations show well-defined filamentary structures in the northeastern region of the remnant, where the brightest optical filaments were located (Lee et al. 2008). These features are interpreted as the recombined neutral gas in an atomic shock (Lee et al. 2008).

Another interesting characteristic of IC 443 is the presence of associated high energy sources. The EGRET GeV source (3EG J0617+2238) was detected overlapping the remnant (Esposito et al. 1996). In addition, TeV gamma-rays from the central region of the remnant, coincident with the maser emission, were recently reported from observations carried out with MAGIC, VERITAS, and Fermi telescopes (Albert et al. 2007; Acciari et al. 2009; Abdo et al. 2010). It has been suggested that the correlation of gamma rays with molecular gas arises from the pion decay of hadronic cosmic rays generated by the interaction of the SNR shock with dense molecular material (Humensky 2008; Abdo et al. 2010).

\section{Observations and data reduction}

The Very Large Array (VLA) ${ }^{1}$ was used in multiple configurations to observe the large SNR IC 443 at 74 and $330 \mathrm{MHz}$. A summary of the observations carried out on various dates between 2005 and 2007 is given in Table 1.

In order to help with the radio frequency interference (RFI) excision and mitigate the effects of bandwidth smearing all the data were acquired in multi-channel continuum mode (64 and 16 channels per polarization at 74 and $330 \mathrm{MHz}$, respectively). At both, 74 and $330 \mathrm{MHz}$, we first performed a bandpass calibration on either 3C 405 (Cygnus A), 3C $147(0542+498)$ or 3C 274 (Virgo A) using publicly available models ${ }^{2}$. The initial antenna-based gain and phase corrections at $74 \mathrm{MHz}$ were estimated from observations of either 3C 405 or 3C 274. For the $330 \mathrm{MHz}$ data, observations of 3C 147 were sufficient for both, flux density and initial phase calibration at this frequency. In all cases the absolute flux scale was set according to the Baars et al. (1977) scale. The NRAO Astronomical Image Processing Software (AIPS) package was used to process all the observations.

Large fields of view are a general characteristic when observing at long radio wavelengths and it is necessary to avoid distortions introduced in the image caused by the non-coplanarity of the baselines of the array. To overcome this, we employed a pseudo-three-dimensional Fourier inversion as implemented in the AIPS task IMAGR, in which the primary beam area of the 74 and $330 \mathrm{MHz}$ data, 11.5 and $\sim 2.5$, respectively, is divided into multiple partially overlapping facets (Cornwell \& Perley 1992). Furthermore, to compensate for time variable ionospheric phase effects we performed successive rounds of self-calibration and imaging to the data from each configuration separately at 74 and $330 \mathrm{MHz}$. Angle-invariant self-calibration (as implemented in the AIPS task CALIB) is generally inadequate for compensating for ionospheric effects across a large field view, particularly for the $74 \mathrm{MHz} B$ and A configuration (Kassim et al. 2007). An exception is for fields dominated by a bright source at the field center, as in the case of IC 443. The final calibrated visibility data from all of the observations listed in Table 1 for

\footnotetext{
1 The Very Large Array of the National Radio Astronomy Observatory is a facility of the National Science Foundation operated under cooperative agreement by Associated Universities, Inc.

2 See http://lwa.nrl.navy.mil/tutorial/ to obtain source models in FITS format.
} 
Table 1. Observational summary.

\begin{tabular}{|c|c|c|c|c|c|c|}
\hline$\overline{\text { Program }}$ & $\begin{array}{c}\text { Observing } \\
\text { dates }\end{array}$ & $\begin{array}{c}\text { VLA } \\
\text { Config. }\end{array}$ & $\begin{array}{c}\text { Bandwidth } \\
(\mathrm{MHz})\end{array}$ & $\begin{array}{l}\text { Integration } \\
\text { time }(\mathrm{h})\end{array}$ & $\begin{array}{c}\begin{array}{c}\text { Synthesized } \\
\text { beam }(\operatorname{arcsec})\end{array} \\
\end{array}$ & $\begin{array}{l}\text { Largest detectable } \\
\text { structure (arcsec) }\end{array}$ \\
\hline \multicolumn{7}{|c|}{$74 \mathrm{MHz}$ Parameters } \\
\hline AG0697 & 2006 Apr. 8, 9, 10 & A & 6.3 & 9 & $25 \times 20$ & 800 \\
\hline AG0735 & 2006 Oct. 27 & $\mathrm{C}$ & 6.3 & 8.35 & $228 \times 214$ & 7500 \\
\hline AG0697 & 2007 Oct. 21 & $\mathrm{AB}$ & 6.3 & 6.85 & $28 \times 30$ & 1300 \\
\hline \multicolumn{7}{|c|}{$330 \mathrm{MHz}$ Parameters } \\
\hline AG0697 & 2005 Sep. 16 & $\mathrm{C}$ & 6.3 & 4.7 & $62 \times 60$ & 1800 \\
\hline AG0697 & 2006 Apr. 8, 9, 10 & A & 1.5 & 9 & $6 \times 6$ & 170 \\
\hline AG0735 & 2006 Oct. 27 & $\mathrm{C}$ & 1.5 & 8.35 & $57 \times 53$ & 1800 \\
\hline AG0735 & 2007 Apr. 15,16 & $\mathrm{D}$ & 6.3 & 5.2 & $238 \times 205$ & 4200 \\
\hline AG0697 & 2007 Oct. 21 & $\mathrm{AB}$ & 1.5 & 6.85 & $22 \times 17$ & 500 \\
\hline
\end{tabular}

each frequency were then combined into a single $u v$ data set, after which a further amplitude and phase self-calibration was performed. For the concatenated $330 \mathrm{MHz}$ data we employed a multi-scale CLEAN algorithm in AIPS, with three different scales sizes. This process is efficient to make high-resolution images that are also sensitive to extended structures. All the resulting facet images were stitched together into one large field using AIPS task FLATN to create a single final image with a synthesized beam of $17^{\prime \prime} .27 \times 15^{\prime \prime} .81, \mathrm{PA}=-24^{\circ}$, which represents an order of magnitude improvement in angular resolution over the $330 \mathrm{MHz}$ image of Braun \& Strom (1986b) and that of Hewitt et al. (2006). The sensitivity achieved in our image after correcting for primary beam attenuation is $1.7 \mathrm{mJy}^{\text {beam }}{ }^{-1}$.

Concerning the observations at $74 \mathrm{MHz}$, we noted that the extended radio emission in the field was more properly imaged using the capability incorporated in the task IMAGR to switch back and forth between the SDI Clean (Steer et al. 1984) and the usual CLEAN deconvolution strategy, depending on the contrast between the brightest residual pixel and the bulk of residual pixels after each major cycle. Following this procedure, the final resolution of the first image of IC 443 obtained at $74 \mathrm{MHz}$ after combining the data sets described in Table 1 and including primary beam corrections is $36^{\prime \prime} .50 \times 31^{\prime \prime} .76$, $\mathrm{PA}=34^{\circ} .71$. The $\mathrm{rms}$ noise level in the $74 \mathrm{MHz}$ image is $30 \mathrm{mJy}^{\text {beam }}{ }^{-1}$.

Ionospheric refraction and self-calibration normally introduce arbitrary position shifts on low-frequency images. These shifts are readily corrected by registering against background small diameter sources whose positions are known from higher frequency observations. We corrected these shifts by measuring several small-diameter sources with respect to their known positions from the NRAO VLA Sky Survey (NVSS) (the latter has an astrometric accuracy better than $1^{\prime \prime}$ in both RA and Dec, Condon et al. 1998). We determined and corrected for a mean positional difference of $0.25 \pm 0.06$ in RA and 5'. $15 \pm 1$ '. 00 in Dec at $74 \mathrm{MHz}$ and $0.08 \pm 0.04$ in RA and 3.' $71 \pm 0 .{ }^{\mathrm{s}} 42$ in Dec at $330 \mathrm{MHz}$.

\section{Results}

\subsection{Low-frequency VLA images of SNR IC 443}

In Fig. 1 we present a close-up view of the radio continuum emission at $74 \mathrm{MHz}$ from IC 443. These observations provide the first subarcminute image of this remnant created at meter wavelengths. The data at $74 \mathrm{MHz}$ are sensitive to smooth structures up to $\sim 125^{\prime}$ in size, larger than the full extent of the SNR, and thus are sensitive to the largest scale structure present in the SNR.

Figure 2 displays with a resolution of $\sim 17^{\prime \prime}$ the new VLA image of the $\sim 2.5$ square degrees field of view centered on the

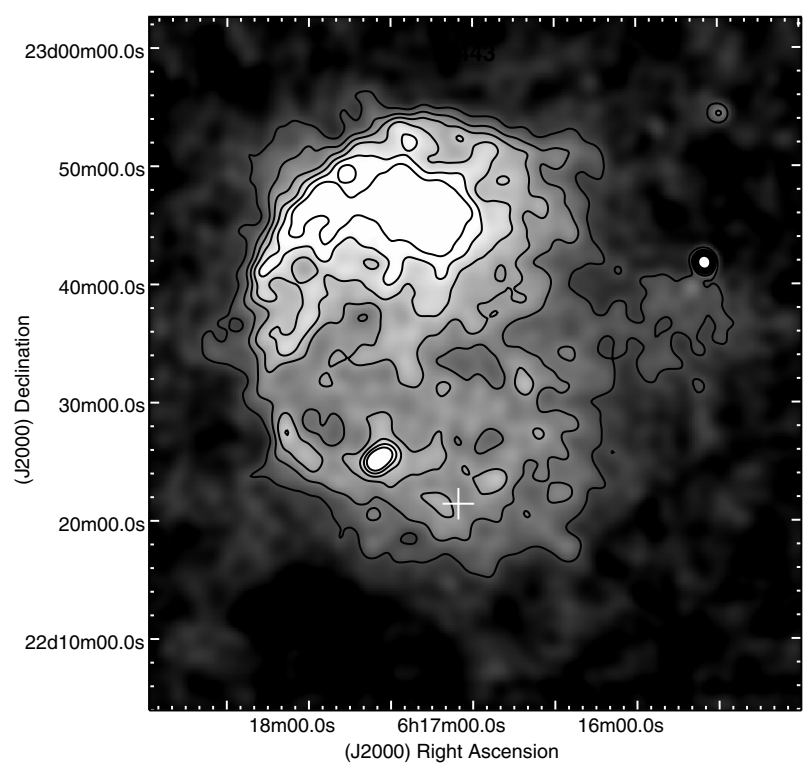

Fig. 1. Radio continuum image of IC 443 at $74 \mathrm{MHz}$. This map has been corrected for the attenuation of the primary beam. The angular resolution of this image was smoothed to a beamsize of $50^{\prime \prime}$. The grayscale is linear ranging from 25 to $250 \mathrm{mJy}^{\text {beam }}{ }^{-1}$ and the contour levels are 72, $120,160,200,240$, and $280 \mathrm{mJy}^{\text {beam }}{ }^{-1}$. The plus symbol marks the position of the compact source CXOU J061705.3+222127. These observations provide the first subarcminute image of the remnant created at meter wavelengths.

remnant observed at $330 \mathrm{MHz}^{3}$. An enlargement showing the detailed $330 \mathrm{MHz}$ total intensity morphology of IC 443 is shown in Fig. 3. The combination of data from the different VLA array configurations ensures that all scales of the radio emission are well represented in the $330 \mathrm{MHz}$ image.

The distinctive morphological characteristics of IC 443, that comprise both the rim-brightened eastern side and the breakout into a region of significantly lower density to the west, were known from past radio observations. For example, a lower resolution WSRT 327 MHz image (Braun \& Strom 1986b, Fig. 5) provides excellent surface brightness sensitivity to the most extended structures. Our $330 \mathrm{MHz}$ image (Fig. 3) is quite complementary, with the higher angular resolution resolving for the first time at such low frequencies small scale structures covering almost the entire extent of the eastern radio shell of the remnant, a region where previous observations have indicated the presence of ionic shocks as well as abundant shocked HI and optical filaments (Lee et al. 2008). It is remarkable that towards the

3 The FITS files of the radio images of IC 443 at 74 and $330 \mathrm{MHz}$ are available online at http://www . iafe.uba.ar/snr/FITS. 


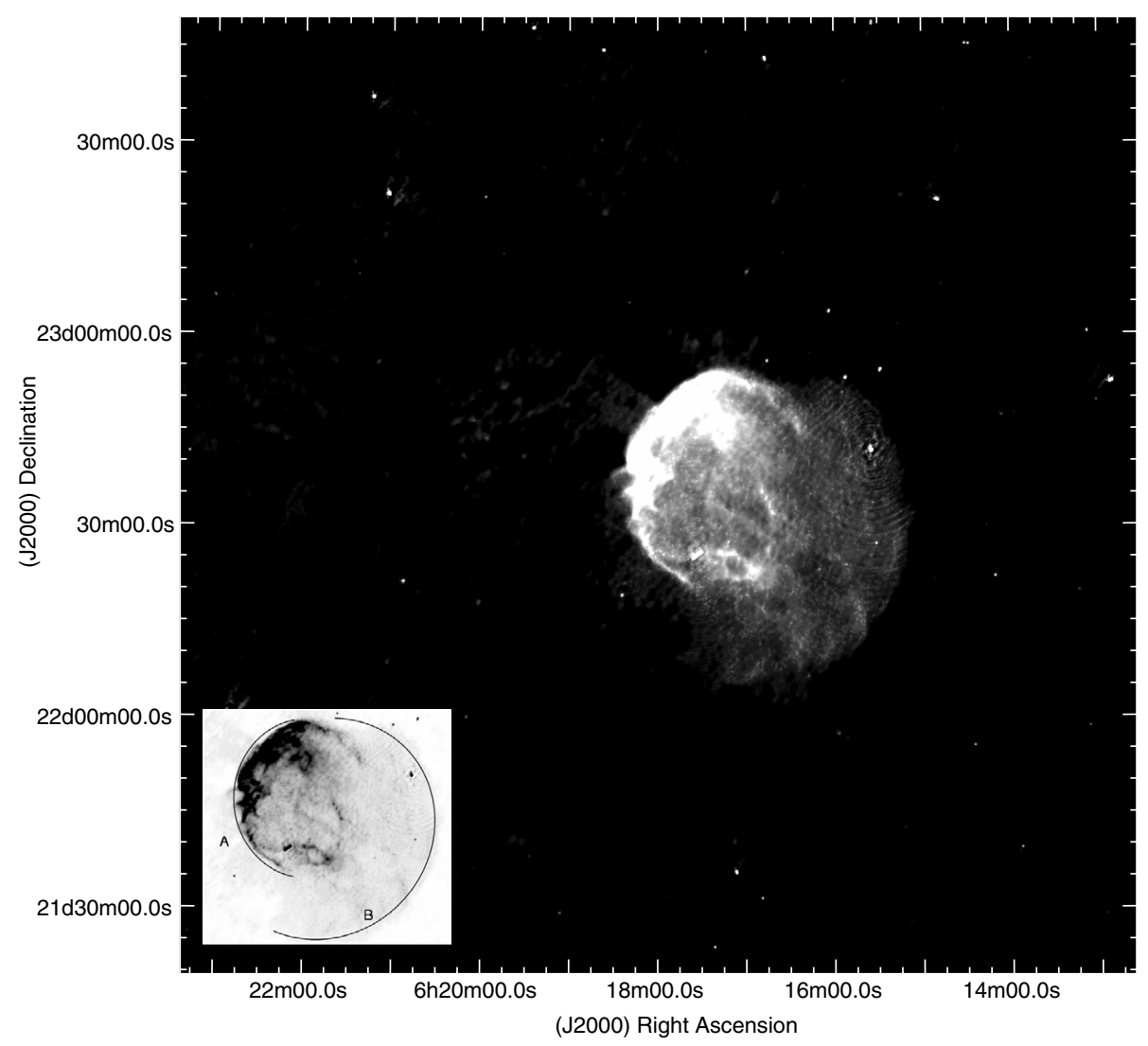

Fig. 2. The image shows the entire field of view of the VLA around the SNR IC 443 at $330 \mathrm{MHz}$. The image displayed includes primary beam correction. The synthesized beam is $17^{\prime \prime} \times 16^{\prime \prime}$ with a position angle of $-24^{\circ}$, and the sensitivity level is $1.7 \mathrm{mJy}^{\mathrm{beam}}{ }^{-1}$. This is the first highfidelity and high-resolution view of the emission from IC 443 ever obtained at low radio frequencies. The inset with the image of IC 443 at $330 \mathrm{MHz}$ is included to help in the location of the bright and weak radio shells referred to in the literature as shells A and B, respectively.

northeast and southeast ends of the eastern shell the brightest emission distribution becomes significantly narrower in appearance. An average diameter of $\sim 35^{\prime}$, or $\sim 15 \mathrm{pc}$ at the assumed distance of $1.5 \mathrm{kpc}$, is measured in our map for the bright radio shell in IC 443 (that, as mentioned in Sect. 1 it is referred to in literature as shell A, and is depicted in the inset in Fig. 2). The low surface brightness emission gradually decreases outwards to the west forming a more uniform radio shell of about $\sim 52^{\prime}$ in diameter or $\sim 23 \mathrm{pc}$ (the so called shell B, Fig. 2), as measured on the new $330 \mathrm{MHz}$ view of the remnant.

Additionally, the $330 \mathrm{MHz}$ image of IC 443 serves to show that even at low radio frequencies part of the east outer border of the remnant is quite structured. It is particularly remarkable the indented morphology of the bright rim at RA $=06^{\mathrm{h}} 18^{\mathrm{m}} 15^{\mathrm{s}}$, Dec $=+22^{\circ} 37^{\prime}$, with tenuous radio synchrotron emission detected ahead of the main shock. Such faint emission is seen as a weak and irregular radio halo that in our $330 \mathrm{MHz}$ image is notable at a mean level of $\sim 4 \sigma$ extending up to $5^{\prime}$ ahead of the bright sharp boundary. It is interesting to note that similar weak, diffuse emission upstream of the main shock is also observed in the cases of Puppis A and W44, along the sides where the expanding blast wave encountered molecular gas (Castelletti et al. 2006, and 2007). A series of small protrusions are also evident along most of the extension of the eastern rim. The largest of these features located at RA $=06^{\mathrm{h}} 18^{\mathrm{m}} 20^{\mathrm{s}}$, Dec $=+22^{\circ} 38^{\prime}$ emerges about $2^{\prime}$ radially from the border of the remnant. These structures can be also seen in the image of IC 443 at $1420 \mathrm{MHz}$ presented by Lee et al. (2008), who refer to them as "spurs". The authors interpret that both, the halo and the "spurs", are likely originated from the interaction of the SNR with the surrounding inhomogeneous ambient medium, although an alternative explanation based on a physical association between IC 443 and the SNR G189.6+3.3 is not entirely ruled out in their work. The low surface brightness emission from $\mathrm{G} 189.6+3.3$, positionally coincident with IC 443 , is clearly detected in our $330 \mathrm{MHz}$ image extending outside the IC 443's eastern shell ${ }^{4}$ near RA = $06^{\mathrm{h}} 18^{\mathrm{m}} 15^{\mathrm{s}}$, Dec $=22^{\circ} 46^{\prime}$.

Towards the center of the SNR, in the southern half, the most remarkable feature is a bright annular filament placed near RA = $06^{\mathrm{h}} 17^{\mathrm{m}} 10^{\mathrm{s}}$, Dec $=+22^{\circ} 24^{\prime}$, which is immersed in the faint and diffuse emission that dominates the inner part of IC 443. Such annular structure forms the central part of a more extended feature commonly referred in the literature as the southern sinuous ridge. The large southern ridge is defined below Dec $\sim+22^{\circ} 35^{\prime}$ at $330 \mathrm{MHz}$ and appears to be an extension of the southeast border of the remnant. Shocked CO gas with broad lines has been detected near this region of the SNR (Cornett et al. 1977; Dickman et al. 1992).

Integrated flux densities estimates for IC 443 were made using the new observations, yielding $S_{74 \mathrm{MHz}}=470 \pm 51 \mathrm{Jy}$ and $S_{330 \mathrm{MHz}}=248 \pm 15 \mathrm{Jy}$. The quoted values have been corrected for the primary beam response and for the contribution of unrelated point sources overlapping the remnant. The uncertainties in the measurements account for the statistical errors as well as the selection of integration boundaries.

4 The radio continuum emission from $\mathrm{G} 189.6+3.3$ is better represented in all its extension in the low resolution image at $327 \mathrm{MHz}$ presented by Braun \& Strom (1986b), displayed with the appropriate contrast to enhance it. 


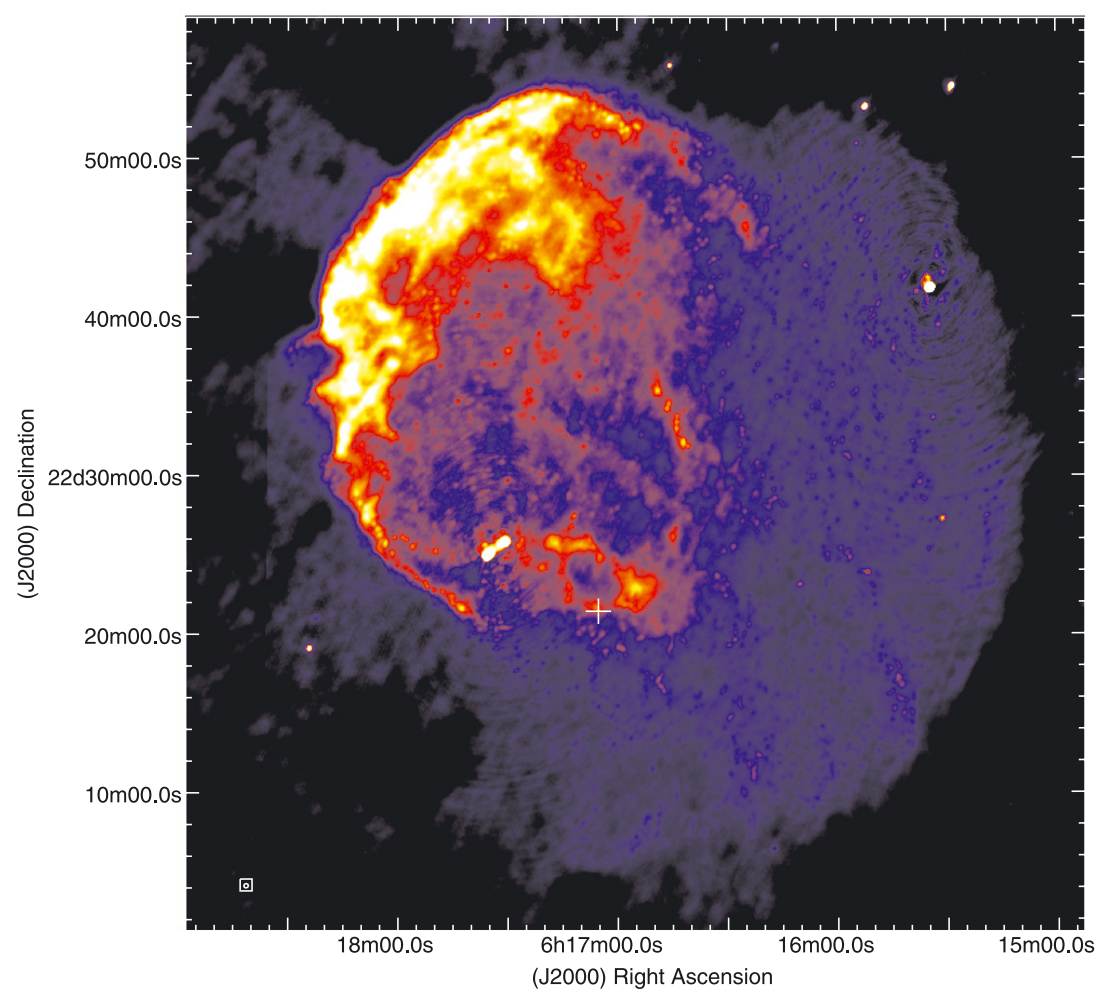

Fig. 3. A color image of the radio continuum emission from IC 443 at $330 \mathrm{MHz}$ constructed using multiple-configuration VLA observations. The brightness range covered by the scale is between 3 and $35 \mathrm{mJy}_{\text {beam }}^{-1}$. The final beam size shown at bottom left is $17^{\prime \prime} \times 16^{\prime \prime}$ at a position angle of $-24^{\circ}$. The noise level is $1.7 \mathrm{mJy}$ beam ${ }^{-1}$ after primary beam correction. The color scale runs between 3 and $45 \mathrm{mJy}^{\text {beam }}{ }^{-1}$. The white plus sign marks the location of the source CXOU J061705.3+222127.

\subsection{The pulsar wind nebula}

The new sensitive image at $330 \mathrm{MHz}$ offers, for the first time, a view of the low frequency counterpart of the low-luminosity plerionic nebula powered by the source CXOU J061705.3+222127 observed both at higher radio frequencies with the VLA and in X-rays with Chandra and XMM-Newton (Olbert et al. 2001; Bocchino \& Bykov 2001; Gaensler et al. 2006).

A close up image of the pulsar wind nebula (PWN) at $330 \mathrm{MHz}$ is displayed in Fig. 4. At $74 \mathrm{MHz}$ the combined effect of the lower sensitivity, poorer angular resolution, and flatter intrinsic spectrum, conspire against its visibility at this low frequency. As in X-rays, at low radio frequencies the source CXOU J061705.3+222127 (marked in Fig. 4 with a plus sign) is placed at the apex of the nebula, although a radio counterpart for the point X-ray source is not detected at $330 \mathrm{MHz}$. The maximum embedded in the radio nebular emission, with an intensity that peaks up to $25 \mathrm{mJy}^{\text {beam }}{ }^{-1}$, lies about 0.25 northeastward from CXOU J061705.3+222127. The cometary shaped nebula extends along its major axis approximately 1.25 behind CXOU J061705.3+222127 and 0.21 ahead of it. The integrated flux density over the entire nebula obtained from our image at $330 \mathrm{MHz}$ is $S_{330 \mathrm{MHz}}^{\mathrm{PWN}}=0.23 \pm 0.05 \mathrm{Jy}$. In addition we estimated the flux density of the PWN at $1420 \mathrm{MHz}$ on the basis of the image of IC 443 presented by Lee et al. (2008) performed by combining VLA observations and single dish data taken from the Arecibo Telescope, obtaining $S_{1420 \mathrm{MHz}}^{\mathrm{PWN}}=0.20 \pm 0.04 \mathrm{Jy}$.

\subsection{Radio and optical emission of IC 443}

Figure 5 illustrates, with a spatial resolution better than $20^{\prime \prime}$, the morphological comparison between the optical and radio emission from IC 443 obtained from the combination in a false

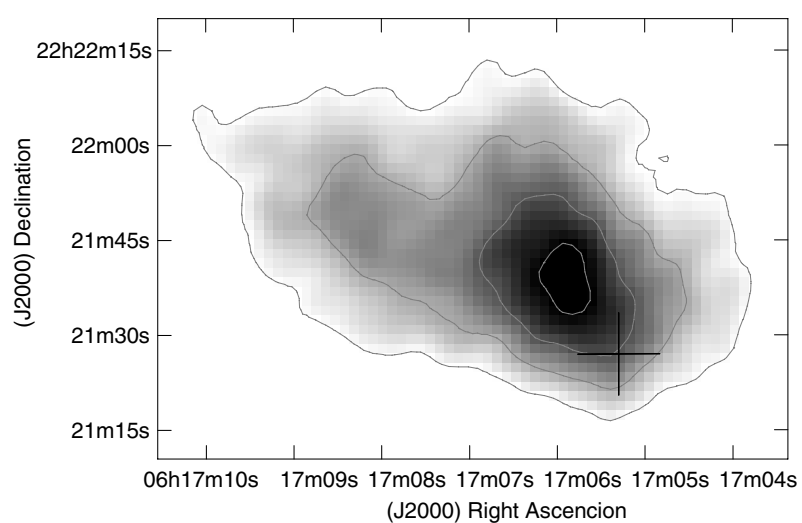

Fig. 4. A close-up image of the nebular emission around CXOU J061705.3+222127 at 330 MHz. The position of CXOU J061705.3+222127 is shown by the plus sign. The grayscale varies from 19 to $25 \mathrm{mJy}^{\text {beam }}{ }^{-1}$. The contour levels on the image are traced at $19,21,23$, and $25 \mathrm{mJy}_{\text {beam }}{ }^{-1}$.

color image of our new VLA observations at $330 \mathrm{MHz}$ (in red) with data from the Second Palomar Observatory Sky Survey (in green). In this figure, features where both spectral bands overlap are shown in yellow.

The optical emission tracing the low density atomic gas reproduces the east-west asymmetry characteristic of the radio total intensity emission as well. The coincidence of the synchrotron-enhanced radio emission with very strong optical filaments observed in $\mathrm{H}_{\alpha}$ and [SII] lines towards the east, suggests that the optical features delineate the position of cooling post-shock ISM gas. Furthermore, near infrared emitting gas observed in this portion of the SNR (see below Fig. 9) together with 


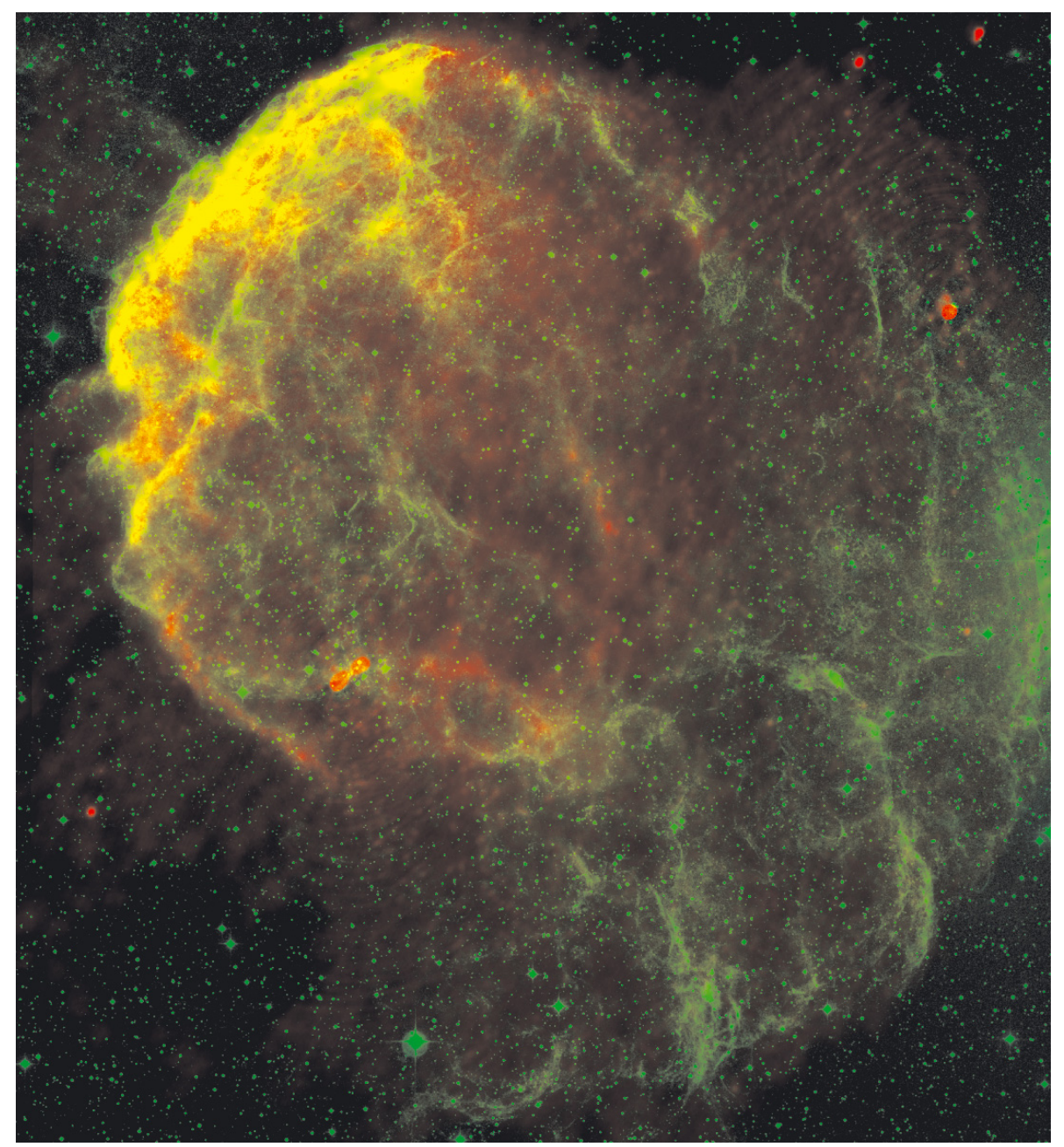

Fig. 5. A high-resolution comparison between radio and optical emission from IC 443 SNR. The green corresponds to optical emission from the Second Palomar Observatory Sky Survey, while in red the $330 \mathrm{MHz}$ radio emission is shown. The yellow regions are areas where emission in both spectral bands overlap.

abundant neutral gas, correlated both in space and velocity with the optical filaments, indicate the presence of radiative shocks propagating into gas of different densities.

The quality of the new $330 \mathrm{MHz}$ image allows us to identify the close radio/optical correspondence in most of the small-scale radio structures observed as extensions from the bright eastern portion of the shell. This is in good agreement with the behavior previously noticed by Lee et al. (2008) using radio continuum observations at $1420 \mathrm{MHz}$.

The southern radio ridge is also mimicked by a quite faint optical counterpart. Absorption due to the molecular gas mainly located in the foreground of the SNR is probably responsible for the observed weakness in the optical emission in this part of IC 443 .

In the breakout region toward the western side of IC 443 only a few local radio enhancements, immersed in faint diffuse radio emission, are observed at the locations of the optical filaments.

\section{Radio spectral properties of SNR IC 443}

\subsection{The spatially resolved spectral index of IC 443}

In this section we analyze the spectral properties of this SNR using the new VLA images presented here at 74 and $330 \mathrm{MHz}$. Estimates of the spectral index variations with position across the remnant require imaging the interferometric data of IC 443 at both frequencies using the same $u v$ coverage. To perform this, we reconstructed the interferometric images by applying appropriate tapering functions to the visibility data at 74 and $330 \mathrm{MHz}$. To show more clearly the main spectral features, avoiding any masking effect from small scale variations, we have chosen a final synthesized beam of $70^{\prime \prime}$. In addition, to avoid any positional offsets, the images were aligned and interpolated to identical projections before calculating spectral indices.

The dependency of the spectral index between 74 and $330 \mathrm{MHz}$ with the position within IC 443 was determined through the construction of a spatially resolved spectral index map, which is shown in Fig. 6. To produce the spectral map the matched images of IC 443 at both frequencies were masked at the $3 \sigma$ level of their respective noise levels. The error in the determination of the spectral index from the map is less than 0.04 in the high flux regions (the east limb of the SNR and some interior filaments) and about 0.1 in the diffuse central emission. The uncertainties increase in the weakest emission regions towards the westernmost part of the remnant due to the lower sensitivity of the image at $74 \mathrm{MHz}$ in this region (see below).

Figure 6 shows good agreement between total intensity features and the spatial distribution of the spectral index. The 74/330 MHz spectral map reveals for the first time the morphology of a very flat spectral component running along the eastern 


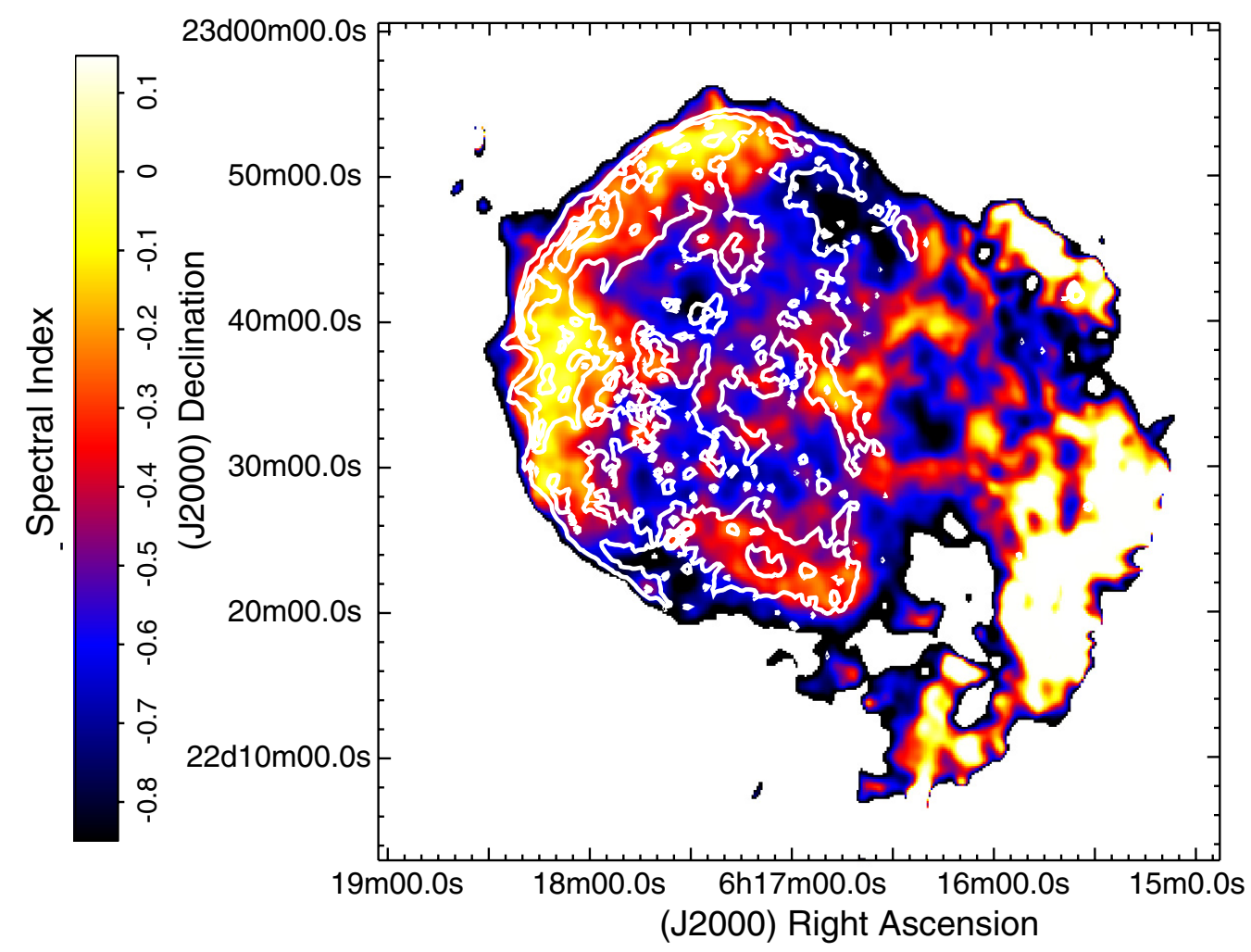

Fig. 6. Spectral index map constructed using VLA observations at 74 and $330 \mathrm{MHz}$ (matched to a common angular resolution of 70"). The 5.5, 15, 26, and $40 \mathrm{mJy}_{\text {beam }}^{-1}$ contours from the $330 \mathrm{MHz}$ image are included to facilitate the comparison between spectral continuum and total power features. To create this map the 74 and $330 \mathrm{MHz}$ images were masked at $3 \sigma$.

side of IC 443 within which the spectral index varies between $\alpha_{74}^{330} \sim-0.05$ and $\sim-0.25$. This flattening towards the brightest filaments (see also Figs. 1 and 3) indicates that some thermal absorption is present, and would become stronger at lower frequencies. It is remarkable that such spectral behavior also has counterparts in the $J$ and $H$ bands as observed by 2MASS (more details for the correlation between local spectral index variation across IC 443 and the IR emission are described below in Sect. 5).

The large-scale diffuse emission in the SNR's interior has a spectrum that is markedly different from the eastern part of the remnant with steeper components ranging from $\alpha_{74}^{330}=-0.6$ to a quite steep value of $\alpha_{74}^{330}=-0.85$, as would be expected under the linear diffusive shock acceleration model for weak shocks with low Mach numbers (Anderson \& Rudnick 1993). The southern sinuous ridge, where most of the interaction with the molecular cloud is taking place, is seen in our spectral index map as a region with $\alpha_{74}^{330}$ varying between $\sim-0.25$ and $\sim-0.5$. These local spectral indices are in good correlation with total intensity features: the brighter synchrotron areas are systematically flatter than the other parts of the ridge. In this case the interpretation of the flattening in the spectrum is different from the case noted above in the east rim. Here, the spectral behavior might be a signature of Fermi shock acceleration at the sites where stronger post compression shock densities, accompanied by higher local Mach numbers, and/or higher magnetic field strength result due to the impact of the SNR blast wave on denser ambient medium (Bell 1978; Anderson \& Rudnick 1993).

Towards the western side of IC 443, which is dominated by diffuse and faint radio emission, the spectral indices are mostly steep $\left(\alpha_{74}^{330} \simeq-0.60\right)$. Although some flatter features are also observed in this region, they are mainly located bordering clipped areas in the map and should not be considered as real features owing to the decrease in the signal-to-noise ratio observed in this region in the VLA $74 \mathrm{MHz}$ image in comparison with the image at $330 \mathrm{MHz}$.

The spectrum calculated for the point-like sources near RA $=06^{\mathrm{h}} 17^{\mathrm{m}} 31^{\mathrm{s}}$, Dec $=+22^{\circ} 25^{\prime} 48^{\prime \prime}$ and $06^{\mathrm{h}} 17^{\mathrm{m}} 36^{\mathrm{s}}$, $+22^{\circ} 24^{\prime} 57^{\prime \prime}$ confirms their extragalactic nature (Braun \& Strom 1986a). A similar result is obtained for the point radio source located at the position RA $\sim 06^{\mathrm{h}} 15^{\mathrm{m}} 34.7^{\mathrm{s}}$, Dec $\sim+22^{\circ} 41^{\prime} 46^{\prime \prime}$.

\subsection{The integrated spectrum of IC 443}

To update the mean spectral index determination for IC 443 , we have included the new integrated flux densities at 74 and $330 \mathrm{MHz}$ in the extensive list of measurements presented in the literature. In Table 2 we list the integrated flux density estimates for the SNR between 10 and $10700 \mathrm{MHz}$. We applied a correction factor over the wide spectral range between 408 and $10700 \mathrm{MHz}$ in order to place each value on the flux scale of Baars et al. (1977), except in some cases for which no information was available on the flux value considered for the primary calibrators.

A plot of the integrated radio continuum spectrum for the SNR IC 443 is shown in Fig. 7. Our new integrated flux measurements at 74 and $330 \mathrm{MHz}$ are indicated by filled circle symbols. From the spectrum it is evident that the flux densities measured at the lowest radio frequencies, and particularly the flux value at $10 \mathrm{MHz}$, lie below the general trend of the data 
Table 2. Integrated flux densities on the SNR IC443.

\begin{tabular}{|c|c|c|c|c|c|}
\hline $\begin{array}{l}\text { Frequency } \\
(\mathrm{MHz})\end{array}$ & $\begin{array}{l}\text { Scaled flux } \\
\text { density (Jy) }\end{array}$ & References & $\begin{array}{c}\text { Frequency } \\
(\mathrm{MHz})\end{array}$ & $\begin{array}{l}\text { Scaled flux } \\
\text { density (Jy) }\end{array}$ & References \\
\hline $10 .$. & $400 \pm 100^{a}$ & Bridle \& Purton (1968) & 430. & $245 \pm 30^{c}$ & Kundu \& Velusamy (1968) \\
\hline $20 \ldots$ & $600 \pm 144^{a}$ & Braude et al. (1969) & 513. & $205 \pm 27^{c}$ & Bondar et al. (1965) \\
\hline 22 . & $615 \pm 75^{a}$ & Roger et al. (1986) & 610. & $215 \pm 32^{c}$ & Dickel \& McKinley (1969) \\
\hline 22.25 . & $535 \pm 65^{a}$ & Roger et al. (1969) & 635. & $179 \pm 18$ & Milne \& Hill (1969) \\
\hline 22.3 . & $529 \pm 36^{a}$ & Guidice (1969) & 740 . & $164 \pm 15^{c}$ & Bondar et al. (1965) \\
\hline 25 . & $630 \pm 132^{a}$ & Braude et al. (1969) & 750 . & $190 \pm 25^{c}$ & Hogg (1964) \\
\hline 26.3 & $600 \pm 48^{a}$ & Viner \& Erickson (1975) & 960. & $196 \pm 24$ & Harris \& Roberts (1960) \\
\hline 26.7 & $561 \pm 33^{a}$ & Guidice (1969) & 960. & $165 \pm 10^{c}$ & Bondar et al. (1965) \\
\hline 33.5 & $582 \pm 37^{a}$ & Guidice (1969) & 1000. & $160 \pm 16^{c}$ & Milne (1971) \\
\hline 34.5 . & $440 \pm 88^{a}$ & Dwarakanath et al. (1982) & 1390. & $177 \pm 15$ & Westerhout (1958) \\
\hline $38 \ldots \ldots$ & $650 \pm 95^{a}$ & Baldwin \& Dewhirst (1954) & $1400 \ldots$ & $170 \pm 20^{c}$ & Hogg (1964) \\
\hline $38 \ldots \ldots$ & $730 \pm 100^{a}$ & Blythe (1957) & $1400 \ldots$ & $146 \pm 18$ & Wanner (1961) \\
\hline $38 \ldots \ldots$ & $460 \pm 46^{a}$ & Williams et al. (1966) & $1410 \ldots$ & $131 \pm 13$ & Milne \& Hill (1969) \\
\hline $38.6 \ldots \ldots$ & $547 \pm 40^{a}$ & Guidice (1969) & $1419 \ldots$. & $130 \pm 13$ & Green (1986) \\
\hline $74 \ldots$ & $470 \pm 51^{a}$ & This work & $1420 \ldots$ & $160 \pm 16^{c}$ & Hagen et al. (1955) \\
\hline 81.5 & $420 \pm 63^{a}$ & Baldwin \& Dewhirst (1954) & $1420 \ldots$ & $138 \pm 15$ & Hill (1972) \\
\hline $81.5 \ldots \ldots$ & $470 \pm 70^{a}$ & Shakeshaft et al. (1955) & $2650 \ldots \ldots$ & $86 \pm 9$ & Milne \& Hill (1969) \\
\hline $83 \ldots \ldots$ & $470 \pm 80^{a}$ & Kovalenko et al. (1994) & $2700 \ldots$ & $104 \pm 15$ & Milne (1971) \\
\hline 102. & $480 \pm 80^{a}$ & Kovalenko et al. (1994) & $3000 \ldots$. & $100 \pm 15^{c}$ & Hogg (1964) \\
\hline $111 \ldots \ldots$ & $440 \pm 80^{a}$ & Kovalenko et al. (1994) & 3125 . & $100 \pm 15^{c}$ & Kuz'min et al. (1960) \\
\hline $151 \ldots \ldots$ & $280 \pm 35^{a}$ & Green (1986) & $4170 \ldots$. & $100 \pm 15^{c}$ & Hirabayashi \& Takahashi (1972) \\
\hline $159 \ldots \ldots$ & $270 \pm 40^{a}$ & Edge et al. (1959) & $5000 \ldots$ & $79 \pm 11$ & Milne (1971) \\
\hline 178. & $210 \pm 42^{a}$ & Bennett (1962) & $5000 \ldots \ldots$ & $85 \pm 13^{c}$ & Kundu \& Velusamy (1969) \\
\hline $195 \ldots \ldots$ & $290 \pm 45^{a}$ & Kundu \& Velusamy (1968) & $6640 \ldots \ldots$ & $70 \pm 15^{c}$ & Dickel (1971) \\
\hline 330 . & $248 \pm 15^{b}$ & This work & $8000 \ldots$ & $90 \pm 18$ & Howard \& Dickel (1963) \\
\hline $400 \ldots \ldots$ & $230 \pm 34^{a}$ & Davies et al. (1965) & $10700 \ldots$ & $60 \pm 5^{c}$ & Kundu \& Velusamy (1972) \\
\hline $400 \ldots \ldots$ & $210 \pm 31^{a}$ & Seeger et al. (1965) & & & \\
\hline $400 \ldots \ldots$ & $251 \pm 8^{a}$ & Kellermann (1964) & & & \\
\hline $408 \ldots \ldots$ & $289 \pm 28$ & Colla et al. (1971) & & & \\
\hline
\end{tabular}

Notes. ${ }^{(a)}$ No correction to Baars et al. (1977) scale was applied. ${ }^{(b)}$ Flux density scale from VLA Calibrator Manual, http://www.aoc.nrao. edu/ gtaylor/csource.html. ${ }^{(c)}$ The correction factor was not available.

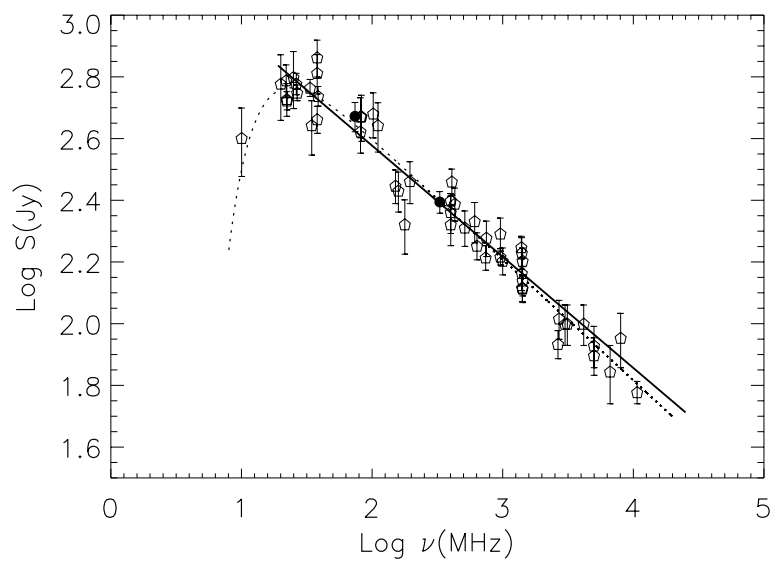

Fig. 7. Radio continuum spectrum for SNR IC 443 obtained from the flux density values listed in Table 2 . The filled circle symbols correspond to the new flux density measurements calculated using the VLA data at 74 and $330 \mathrm{MHz}$ presented in this work, the open symbols are for radio observations previously published and, where possible, brought onto the flux density scale of Baars et al. (1977). Solid line represents the linear fit to the flux density values excluding that at $10 \mathrm{MHz}$, which produces a spectral index $\alpha=-0.36 \pm 0.02\left(S \propto v^{\alpha}\right)$. Dotted line shows a fit to all of the plotted values if absorption were present (Eq. (1)), which yields a spectral index $\alpha=-0.39 \pm 0.01$.

suggesting the presence of thermal absorption along the line of sight. In order to fix the integrated spectral index of IC 443 we first use a single power law spectrum to fit the spectrum well down to our lowest measurement at $74 \mathrm{MHz}$, excluding the flux density value at $10 \mathrm{MHz}$ (represented as a solid line in Fig. 7). A weighted fit produces a spectral index $\alpha=-0.36 \pm 0.02$ $\left(S_{v} \propto v^{\alpha}\right)$. This result agrees very well within the error limits with the previous estimates presented by Erickson \& Mahoney (1985, and references therein). If we consider the lowest frequencies observations, these measurements can be fit with a power law plus an exponential turnover using Eq. (1) (indicated by the dotted line in Fig. 7)

$$
S_{v}=S_{330}\left(\frac{v}{330 \mathrm{MHz}}\right)^{\alpha} \exp \left[-\tau_{330}\left(\frac{v}{330 \mathrm{MHz}}\right)^{-2.1}\right]
$$

here, $330 \mathrm{MHz}$ is a reference frequency at which an integrated flux density $S_{330}$ and an optical depth $\tau_{330}$ are measured, $\alpha$ represents the non-thermal integrated spectrum, which is assumed to be constant throughout the radio band. We have made a weighted fit of the distribution of data points over four decades in frequency and find for the whole SNR a single radio spectral index $\alpha=-0.39 \pm 0.01$, and an average optical depth $\tau_{330}=(7 \pm 1) \times 10^{-4}$. The free-free continuum optical depth at $10 \mathrm{MHz}$ derived from the relation $\tau_{10}=\tau_{330}[10 / 330]^{-2.1}$ is $\tau_{10}=1.07$, while the optical depth at $74 \mathrm{MHz}$ is $\tau_{74}=0.02$. Our results indicate that although the absorption becomes significantly stronger at $10 \mathrm{MHz}$ this effect is negligible at $74 \mathrm{MHz}$. In addition, we note that the spectral index produced by considering free-free absorption is consistent with that derived with a power law. Such a result is not surprising given the results of Sect. 4.1, 




Fig. 8. Radio continuum spectrum for the pulsar wind nebula (PWN) around CXOU J061705.3+222127 in the SNR IC 443. A weighted fit to the data with a single power law function $\left(S \propto v^{\alpha}\right)$ yields a spectral index $\alpha=-0.04 \pm 0.05$. The filled circles symbols are from the flux density estimates at 330 and $1420 \mathrm{MHz}$ presented in the current work, while the open symbols are from Olbert et al. (2001).

that clearly indicated the presence of thermal absorption, though not at a level to impact the integrated flux at $74 \mathrm{MHz}$. Future measurements at intermediate frequencies (e.g. $\sim 30 \mathrm{MHz}$ ) are needed to understand if the turnover inferred from the $10 \mathrm{MHz}$ measurement is related to the subtle absorption revealed in the spectral index analysis presented in Sect. 4.1.

Additionally we re-calculate the spectrum of the pulsar wind nebula in IC 443. To analyze the global spectrum of the PWN in IC 443 we combine the new flux densities estimated at 330 and $1420 \mathrm{MHz}$ with data taken from Olbert et al. (2001) at 1460, 4860, and $8460 \mathrm{MHz}$. The radio spectrum including all these measurements is shown in Fig. 8. The larger error bars for the lower frequency radio data reflect the difficulty in separating the nebular emission from its surroundings. A weighted fit to all the flux densities produces a spectral index for the PWN of $\alpha=$ $-0.04 \pm 0.05$, which is zero within the uncertainty in the fit itself. This result is similar to that obtained by Olbert et al. (2001).

\section{Radio spectral index and the near infrared emission}

Based on our accurate, spatially resolved radio continuum $74 / 330 \mathrm{MHz}$ spectral map, we have investigated the correlation between radio spectral features and the near infrared emission (NIR). Figure 9a shows two 74/330 $\mathrm{MHz}$ spectral index contours (traced at $\alpha_{74}^{330}=-0.05$ and $\alpha_{74}^{330}=-0.25$ ) enclosing the east rim where we found regions with very flat spectrum in IC 443 superposed onto the NIR detected in the $J(1.25 \mu \mathrm{m})$, $H(1.65 \mu \mathrm{m})$, and $K_{\mathrm{s}}(2.17 \mu \mathrm{m})$ bands as taken from the Two Micron All Sky Survey (2MASS) (Rho et al. 2001). The 2MASS image of IC 443 shows the dramatic contrast in near infrared color between the east rim and the southern portion of the remnant. In the color representation of the infrared emission, blue traces the $J$-band flux, while the infrared data in the $H$ and $K_{\mathrm{s}}$ are shown in green and red, respectively; white thus enlightens regions where all the three IR bands overlap. To facilitate the comparison, Fig. $9 \mathrm{~b}$ displays the $330 \mathrm{MHz}$ continuum image of IC 443 with a grayscale selected to emphasize the brightest radio emission.

An impressive agreement is observed in location, size and shape between the NIR emission detected in the $H$ and $J$ bands and the flattest spectral feature as traced by the $\alpha_{74}^{330}$ contours along the eastern edge of IC 443. This correspondence begins in the northernmost part of the remnant and extends down to positions near Dec $\sim+22^{\circ} 25^{\prime}$. From Fig. $9 \mathrm{~b}$ it is also notable that the brightest radio synchrotron emission perfectly matches the bright emission in the NIR bands. As noticed by Rho et al. (2001), at this site the predominant constituent of the emission in the $J$ and $H$ bands is the [FeII] line, with a minor contribution from other multi-ionized species like [NeII], [NeIII], [SiII], [SIII], etc. Rho et al. (2001) proposed a model in which the infrared emission from the ionized species in the east bright radio limb of IC 443 comes from shattered dust produced by a fast dissociating J-type shock. The present accurate comparison between radio spectral indices and IR emission confirms this model. In effect, the passage of a dissociative shock through a molecular cloud not only dissociates molecules but also ionizes the atoms. Such collisional ionization is responsible for the thermal absorbing electrons that produce the peculiar very flat spectrum areas observed all along the eastern border of IC 443. This interpretation is in agreement with studies based on $\mathrm{CO}$ and X-ray observations which conclude that the large molecular cloud complex is located in front of IC 443 (Cornett et al. 1977; Troja et al. 2006). Evidence for a similar situation has also been observed in the ringlike morphology of 3C 391 (Brogan et al. 2005), another SNR known to be interacting with a molecular cloud.

Towards the interior of the bright eastern shell, the spectral index gradually steepens with position in coincidence with a decrease in the intensity of the radio emission. Widely distributed $K_{\mathrm{s}}$ band emission is observed in the 2MASS image in this part of the remnant, which was proposed to delineate $\mathrm{H}_{2}$ shocked gas from the region interacting with the adjacent molecular cloud.

In contrast to the excellent agreement between the ionic emitting gas and the flattest spectrum features found in the eastern bright limb, no much obvious correspondence is observed in the southern part of IC 443 with the exception of a spectral component located at the northern extreme of the ridge, near RA $=06^{\mathrm{h}} 16^{\mathrm{m}} 45^{\mathrm{s}}$, Dec $=22^{\circ} 35^{\prime}$. This poor IR/radiospectrum correspondence is consistent with the hypothesis proposed before, in which the flat spectrum features in this part of IC 443 have a non-thermal origin.

In the remainder of this section we attempt to infer, using our new measurements at low radio frequencies, the physical properties of the area of thermal absorption seen in Fig. 9a spatially coincident with the ionized fine-structure line emitting atoms, i.e. the eastern half of the SNR. At radio wavelengths, the emission measure (EM) is given by,

$\mathrm{EM}=6.086 \times 10^{-6} a\left(T_{\mathrm{e}}, v\right)^{-1} v^{2.1} \tau_{v} T_{\mathrm{e}}^{1.35} \mathrm{~cm}^{-6} \mathrm{pc}$,

where $a\left(T_{\mathrm{e}}, v\right)$ is the Gaunt factor assumed to be 1 , a correct value for the range of astrophysical quantities involved in our calculations; $v$ and $\tau_{v}$ are the frequency measured in $\mathrm{MHz}$ and the free-free optical depth, respectively, and $T_{\mathrm{e}}$ is the electron temperature in $\mathrm{K}$ of the intervening ionized gas. By measuring the relative strengths of the [FeII] lines observed in the near and mid infrared emission, Rho et al. (2001) conclude that the emitting region behind the J-type shocks as observed in the $J$ and $H$ bands of the 2MASS has a temperature of $12000 \mathrm{~K}$. Although not quantified, the authors recognize a large uncertainty associated with this magnitude as a consequence of different beam size and possibly different filling factors in their measurements. If we assume that $T_{\mathrm{e}}$ is in a reasonable range between $8000-12000 \mathrm{~K}$ (which includes the temperature as estimated from the IR observations) and use the optical depth derived from our radio 

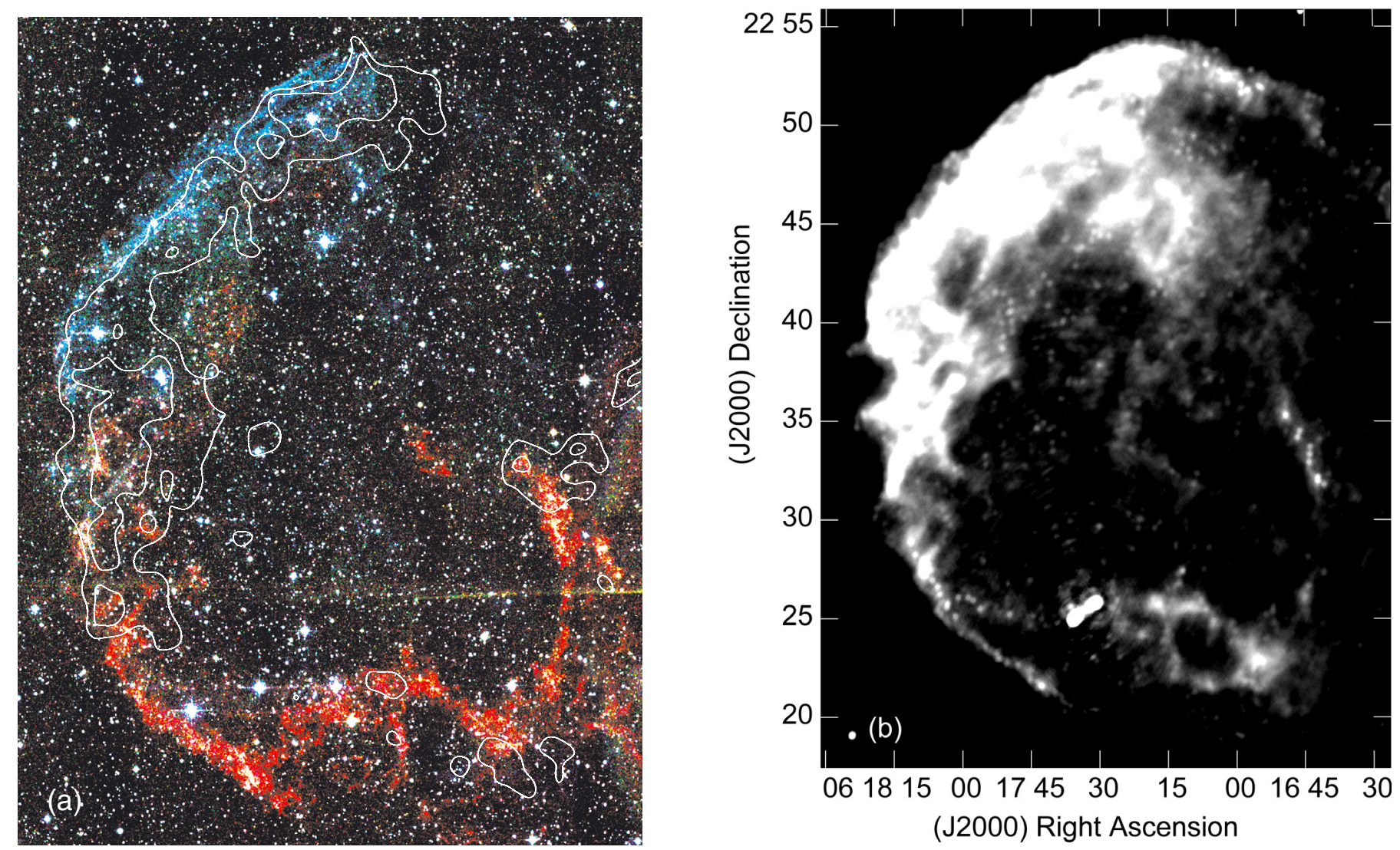

Fig. 9. a) A color representation of the near-infrared emission observed with 2MASS in the $J$ (in blue), $H$ (in green), and $K_{\mathrm{s}}$ (in red) bands (Rho et al. 2001). The overlaid contours trace the flattest-spectrum radio structures of the SNR IC443 between 74 and $330 \mathrm{MHz}$ at $\alpha_{74}^{330}=-0.05$ and -0.25 . b) $330 \mathrm{MHz}$ image of IC 443 showing the locations of the brightest regions of the remnant.

study particularly for this region where the thermal absorption is stronger $\left(\tau_{74} \sim 0.3\right)$ we obtain an EM between approximately $2.8 \times 10^{3}$ and $5.0 \times 10^{3} \mathrm{~cm}^{-6} \mathrm{pc}$ for the eastern rim. By combining this emission measure with the postshock electron density, we can roughly calculate the thickness of the molecular gas layer that has been dissociated and ionized by the SNR shock front. If we assume an electron density of $n_{\mathrm{e}} \sim 500 \mathrm{~cm}^{-3}$ as estimated by Fesen \& Kirshner (1980) and Reach \& Rho (2000) on the basis of forbidden [FeII] lines, we conclude that the dissociation and ionization processes took place in a thin screen of about 3.4 to $6.0 \times 10^{16} \mathrm{~cm}(\sim 0.01-0.02 \mathrm{pc})$. This is a small path compared with the transverse dimensions over which thermal absorption is observed, but is a lower limit if the ionized gas is clumped.

\section{Comparison with the molecular distribution}

In Fig. 10 we present a comparison between our VLA $330 \mathrm{MHz}$ image and new ${ }^{12} \mathrm{CO}(J=1-0)$ data presented by Zhang et al. (2010) on the basis of new observations carried out with the telescope of the Purple Mountain Observatory in China (main beam size of $50^{\prime \prime} \times 54^{\prime \prime}$, velocity resolution of $0.37 \mathrm{~km} \mathrm{~s}^{-1}$, and $\mathrm{rms}$ noise level of $0.1-0.3 \mathrm{~K}$ at a velocity resolution of $\sim 0.2 \mathrm{~km} \mathrm{~s}^{-1}$ ). The contours superposed on the radio emission depict the $\mathrm{CO}$ emission integrated in the range between -10 and $-1 \mathrm{~km} \mathrm{~s}^{-1}$, which includes the systemic velocity of IC 443 . As described before, the molecular material is preferentially located in the center of the remnant extending in the southeast-northwest direction. The spatial distribution of the molecular gas across IC 443 is clearly non-uniform. Earlier observations have identified the presence of various clumps of molecular gas with broad line widths, as expected from the interaction with the supernova shock front (clumps labeled from $\mathrm{A}$ to $\mathrm{H}$ in the nomenclature of Dickman et al. 1992).

On the basis of the new image at $330 \mathrm{MHz}$ it is possible to recognize details previously unnoticed in the spatial comparison between the radio emission and the molecular gas. In Fig. 10 we display the regions where good correlation between radio features and molecular gas distribution is observed. Particularly noticeable is Fig. 10b where it is apparent that the indentation of the eastern border in radio occurs near a region where a significant enhancement in the $\mathrm{CO}$ emission is detected. The molecular complex is transverse to the radio indentation with the maximum of the $\mathrm{CO}$ emission shifted to the southwest in at least $3^{\prime}$ from the border of the SNR (in the region of the molecular clump E). It is possible that the singular indentation has formed as the result of the supernova shock front wrapping around a dense clump. Also, Fig. 10c shows the presence of a concentration in the CO emission around RA $=06^{\mathrm{h}} 17^{\mathrm{m}} 16^{\mathrm{s}}$, Dec $=+22^{\circ} 25^{\prime} 40^{\prime \prime}$ (molecular clump B), that matches a local maximum in the radio emission. The morphological matching between the radio synchrotron emission and the molecular gas is especially remarkable at the northern extreme of the southern ridge of IC 443 near $\mathrm{RA}=06^{\mathrm{h}} 16^{\mathrm{m}} 45^{\mathrm{s}}$, Dec $=+22^{\circ} 34^{\prime} 00^{\prime \prime}$ (as shown in Fig. 10d), where the molecular contours, delineating high density gas in the region of the clump $\mathrm{G}$, are observed enclosing the bright radio emission.

We also searched for spectral evidence of shock/cloud interaction. Figure 11 displays an overlay of the ${ }^{12} \mathrm{CO}(J=$ $1-0)$ integrated emission contours with the radio spectral index 

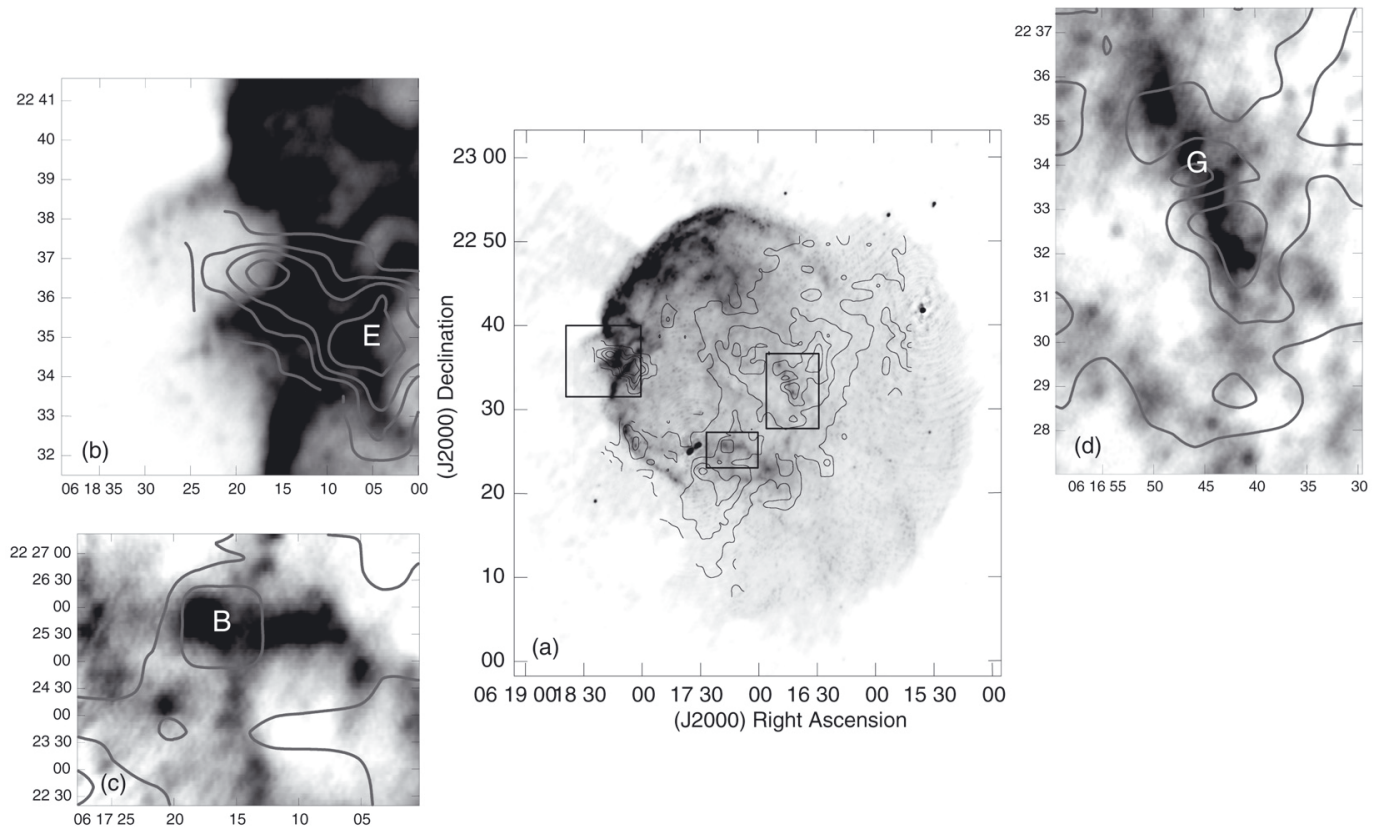

(J2000) Right Ascension

Fig. 10. A comparison of the radio continuum emission of IC 443 and the ${ }^{12} \mathrm{CO}(J=1-0)$ distribution in the SNR region. The grayscale representation corresponds to the new VLA $330 \mathrm{MHz}$ image, while the superposed contours trace CO integrated emission from -10 to $-1 \mathrm{~km} \mathrm{~s}^{-1}$ as taken from Zhang et al. (2010). Close-ups of three interesting areas are displayed around the center image, with the CO contours overlapping. The white letter in each panel corresponds to the designation of the molecular clump by Dickman et al. (1992).

distribution calculated between 74 and $330 \mathrm{MHz}$. The CO molecular gas seen in projection onto the plane of the sky, overlaps the only flat spectral region observed in the interior of IC 443. Various small components with a spectrum appreciably flatter than the surrounding synchrotron plasma are observed distributed nearby or in coincidence with local higher density gas as traced by the ${ }^{12} \mathrm{CO}$ contours along the bright southern ridge and towards the northwest, suggesting that these features must be regions where strong shocks encountered denser material (as discussed by Anderson \& Rudnick 1993). In the eastern periphery the situation is different. The very flat spectrum component extends over an area considerably larger than the molecular cloud, precisely because in this region, as shown in Sect. 5, most of the molecules were dissociated and ionized, absorbing the radio emission at low frequencies.

In Fig. 11 we have identified with open white diamonds the regions with $\mathrm{OH}(1720 \mathrm{MHz})$ maser emission as detected by Hewitt (2009). In the case of SNR W28, Dubner et al. (2000) demonstrated a clear correspondence between regions of flat spectral index and $\mathrm{OH}$ maser emission. We search for a similar correlation in IC 443. From Fig. 11 we conclude that there is not a simple association between maser locations and spectral index features but rather both flat and steep components are observed near masers areas. This fact can be compared with previous results, which suggest that maser regions in IC 443 arise from regions with different shock geometry: a shock mostly propagating towards the line of sight in the southern $\mathrm{OH}$ maser emission and a transverse shock in the westernmost $\mathrm{OH}$ emitting region (Claussen et al. 1997; Hoffman et al. 2003; Hewitt 2009). We find that the $\mathrm{OH}$ maser area with transverse shock $\left(\mathrm{RA}=06^{\mathrm{h}} 16^{\mathrm{m}} 43^{\mathrm{s}} \cdot 6, \mathrm{Dec}=+22^{\circ} 32^{\prime} 36^{\prime \prime} .7\right)$ correlates well with flat spectral index $\left(\alpha_{74}^{330}=-0.4\right)$, and one of the $\mathrm{OH}$ regions where the shock is tangential to the line of sight $\left(\mathrm{RA}=06^{\mathrm{h}} 17^{\mathrm{m}} 29^{\mathrm{s}} \cdot 29\right.$, Dec $\left.=+22^{\circ} 22^{\prime} 42^{\prime \prime} .5\right)$ is associated with a steep spectrum $\left(\alpha_{74}^{330}=-0.8\right)$. The fact that

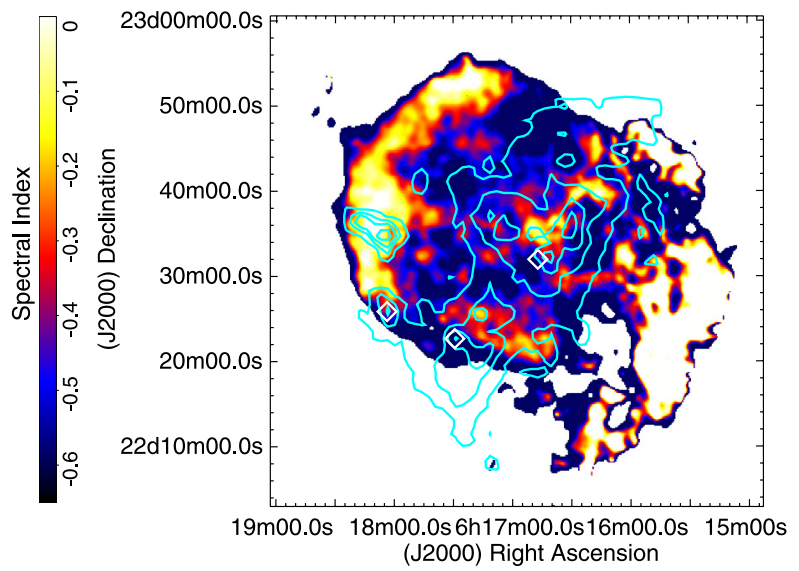

Fig. 11. A comparison of the spectral index distribution with the molecular emission. The color representation corresponds to the spatially resolved spectral index map between 74 and $330 \mathrm{MHz}$, while the overlaid contours trace the ${ }^{12} \mathrm{C} 0(J=1-0)$ emission integrated between -10 and $-1 \mathrm{~km} \mathrm{~s}^{-1}$ (Zhang et al. 2010). The positions where $\mathrm{OH}(1720 \mathrm{MHz})$ maser emission were detected are indicated with open white diamonds (Hoffman et al. 2003; Hewitt 2009).

flat spectrum emission $\left(\alpha_{74}^{330}=-0.4\right)$ is observed towards the easternmost $\mathrm{OH}$ maser area with tangential shock $(\mathrm{RA}=$ $06^{\mathrm{h}} 18^{\mathrm{m}} 3^{\mathrm{s}} .67$, Dec $=+22^{\circ} 25^{\prime} 53^{\prime \prime} .4$ ) can be explained because in this region thermal electrons mask the intrinsic SNR spectral effects.

\section{Comparison with $\mathrm{TeV}$ emission}

Figure 12 compares the $\mathrm{TeV}$ gamma-ray significance contours obtained from VERITAS observations (Acciari et al. 2009) along with the $74 / 330 \mathrm{MHz}$ spectral index map presented in Fig. 6 (Fig. 12a), with the total intensity features of IC 443 at 

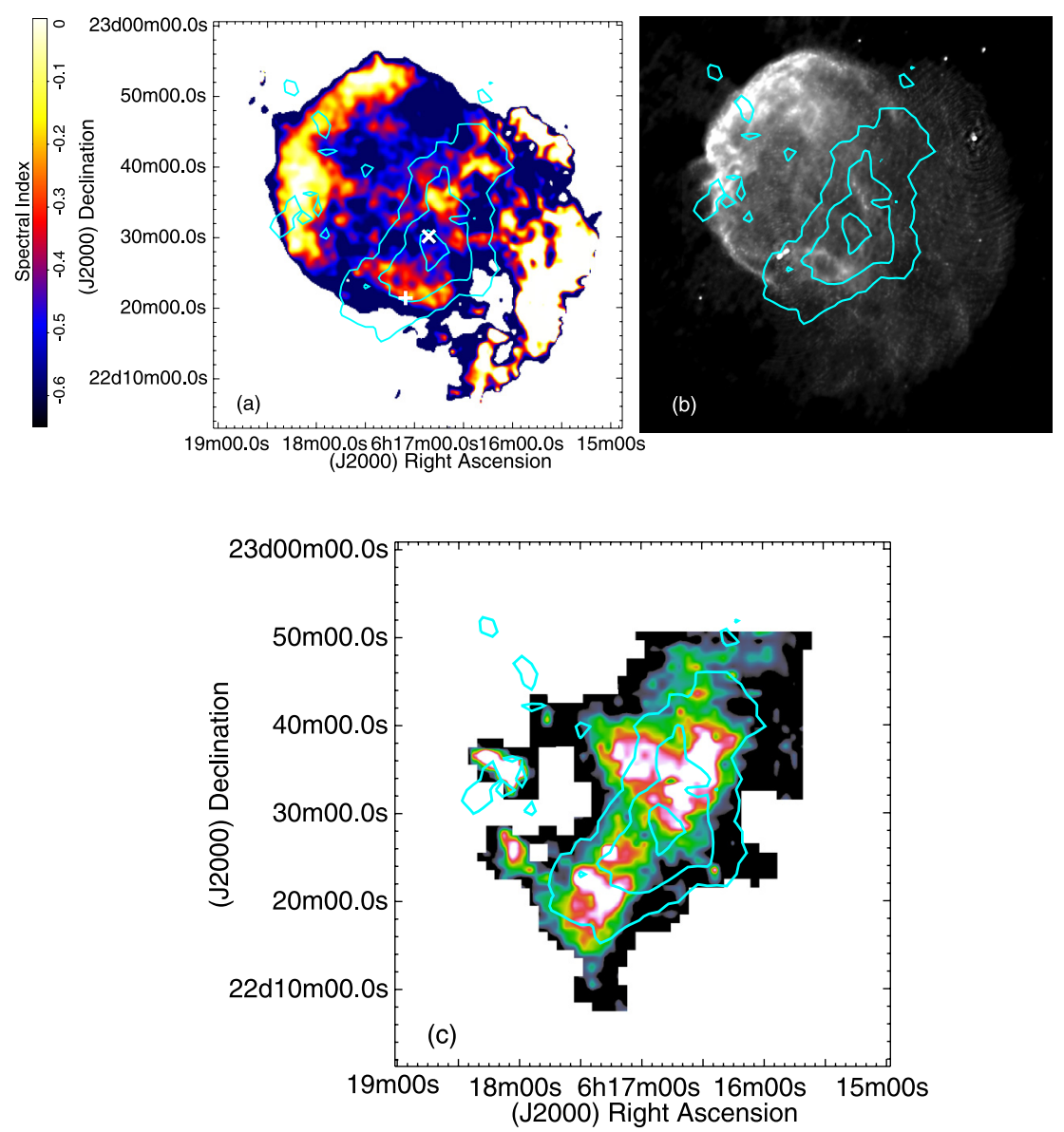

Fig. 12. Very high energy gamma-rays contours as taken from VERITAS (Acciari et al. 2009) superposed with a) the 74/330 MHz spectral index map presented in Fig. 6, b) radio continuum emission at $330 \mathrm{MHz}$, and c) ${ }^{12} \mathrm{C} 0(J=1-0)$ integrated emission from -10 to $-1 \mathrm{~km} \mathrm{~s}{ }^{-1}$ tracing the molecular cloud interaction with IC 443 (Zhang et al. 2010). The white plus sign and the white cross in a) mark the positions of the source CXOU J061705.3+222127 and the centroid of the TeV source VER J0616.9+2230, respectively.

$330 \mathrm{MHz}$ (Fig. 12b), and with the ${ }^{12} \mathrm{CO}(J=1-0)$ molecular gas distribution (Fig. 12c). In Fig. 12a the white plus sign marks the position of the source CXOU J061705.3+222127, while the white cross indicates the centroid of the TeV source VER J0616.9+2230.

Very high energy (VHE) gamma-ray emission roughly extends along the southeast-northwest axis, overlapping most of the central region of the remnant. On the basis of the available statistics, no correlation can be demonstrated between the radio spectrum or radio features with the $\mathrm{TeV}$ emission region. On the contrary, it is remarkable the morphological coincidence between the $\mathrm{TeV}$ emission and the molecular gas distribution (Fig. 12c), at least up to the level that the available TeV statistic permits us to confirm. The interpretation of this striking correspondence is beyond the scope of this paper.

\section{Conclusions}

In this work we report on new full-synthesis imaging of the Galactic SNR IC 443 generated from multiple-configuration VLA observations at 74 and $330 \mathrm{MHz}$. These high-fidelity images constitute the best angular resolution, low-frequency radio study published to date on this classic remnant. Based on these new data we measured integrated flux densities for this object of $S_{74 \mathrm{MHz}}=470 \pm 51 \mathrm{Jy}$ and $S_{330 \mathrm{MHz}}=248 \pm 15 \mathrm{Jy}$. On the basis of these new total flux density measurements at
74 and $330 \mathrm{MHz}$ together with previously published values, we recalculated the integrated spectrum of IC 443 . We have made this analysis, for the first time, taking into account that the spectrum of IC 443 has a reported turnover at the lowest radio frequencies. The fit over a wide frequency range produces a radio spectral index $\alpha_{10 \mathrm{MHz}}^{10700 \mathrm{MHz}}=-0.39 \pm 0.01$ with free-free thermal optical depth $\tau_{330} \sim 7 \times 10^{-4}$ (or $\tau_{74} \sim 0.02$ ). If the measurement at $10 \mathrm{MHz}$ is excluded, we note that $\alpha$ is relatively unchanged from that inferred from a single power law fit $\left(\alpha_{10 \mathrm{MHz}}^{10700 \mathrm{MHz}}=-0.36 \pm 0.02\right)$.

Based on the combination of the new images at 74 and $330 \mathrm{MHz}$, we investigated spectral changes with position across IC 443 and related them to the spatial characteristics of the radio continuum emission and of the surrounding interstellar medium. For the first time we spatially resolved the flattest spectrum region over the SNR (with very flat indices down to $\alpha_{74}^{330} \sim-0.05$ ) along the brightest rim on the eastern side of the remnant. The radio spectrum steepens towards the more diffuse component in the interior of IC 443 with $\alpha_{74}^{330}$ between -0.6 and -0.85 , consistent with those expected from linear diffusive shock acceleration processes. Finally, another region with non-uniform flattening in the spectrum is identified near the southern ridge of IC 443 . We investigated the connection between these spatial spectral variations and IR and molecular emission distribution.

The comparison with 2MASS NIR data underscored an impressive coincidence between the eastern radio flattest spectrum 
region and NIR ionic lines which account for most of the infrared emission observed towards this part of IC 443. Based on the presence of this IR emission, which confirms the existence of a strong J-type shock dissociating molecules and ionizing atoms, we conclude that the most likely explanation for the flattest spectrum observed here, is that it is produced by freefree absorption at $74 \mathrm{MHz}$ along the line of sight (with $\tau_{74}$ up to $\sim 0.3$ ). From the physical parameters of the IR emitting gas together with our new radio spectral study, we conclude that the observed thermal absorption takes place in a very thin layer (width $\sim 0.02 \mathrm{pc}$ ) that extends all along the eastern border. Such a thin layer would be the product of the dissociating/ionizing action of the SNR shock over the adjacent molecular gas. It is important to point out that the low optical depths derived for the whole SNR $\left(\tau_{330} \sim 7 \times 10^{-4}\right.$ and $\left.\tau_{74} \sim 0.02\right)$ indicate that the 74 and $330 \mathrm{MHz}$ integrated emission is not significantly attenuated, highlighting that the thermal absorption inferred from the spatially resolved spectral index map is a relatively subtle and localized effect. Our result only represents the second case, following 3C 391 (Brogan et al. 2005), of spatially resolved thermal absorption delineating the interaction of an $\mathrm{SNR} /$ molecular cloud shock boundary. Moreover it confirms such phenomena as common and a rich area of investigation for future, low-frequency studies of Galactic complexes.

On the other hand, from the molecular studies it is known that the southern ridge, where the second region with flat spectrum was identified, is the site in which the most complex interaction between the SNR shock and the external molecular cloud is occurring. We used Zhang et al. (2010)'s observations of the ${ }^{12} \mathrm{CO}(J=1-0)$ molecular gas towards IC 443 to analyze correspondences between these data and our new low radio frequency observations. The correlation of the emission at $330 \mathrm{MHz}$ with the new ${ }^{12} \mathrm{CO}$ observations revealed spatial irregularities in the denser molecular gas that are well associated with features of bright emission in radio and good agreement between the flat radio spectrum region towards the south and molecular emission. We conclude that here the flat radio spectrum is predominantly a signature of shock acceleration in a region with strong postshock densities and enhanced magnetic fields produced after the interaction of the blast wave with dense ambient medium.

Furthermore, from the comparison of the molecular environment mapped by ${ }^{12} \mathrm{CO}$ data with the local variations of the spectral index, we find evidence that the shocked molecular gas, as illuminated by the $\mathrm{OH}(1720 \mathrm{MHz})$ maser emission, is coupled with a flattening in the radio spectral index in the locations where the shock is transverse to the line of sight, while a steep spectrum is observed in a $\mathrm{OH}$ maser region in which the shocks are propagating along the line of sight.

From the new images, we also analyzed the pulsar wind nebula around the source CXOU J061705.3+222127 in IC 443. Based on the new VLA image at $330 \mathrm{MHz}$ we have derived a flux density $S_{330 \mathrm{MHz}}^{\mathrm{PWN}}=0.23 \pm 0.05$. We have also measured the flux density of the PWN in the radio continuum image at $1420 \mathrm{MHz}$ presented by Lee et al. (2008) obtaining $S_{1420 \mathrm{MHz}}^{\mathrm{PWN}}=$ $0.20 \pm 0.04$. From the combination of these data and measurements taken from the literature we derived an integrated radio spectrum $\alpha_{330}^{8460} \sim 0.0$ for the PWN.

In addition, on the basis of available VERITAS statistics we compared the $\mathrm{TeV}$ emission with the ${ }^{12} \mathrm{CO}$ distribution, finding an excellent morphological correlation between the high energy emission and the distribution of the molecular gas. No correspondence was found, however, between the gamma-ray emission as observed by VERITAS and radio spectral or intensity features in radio. A future paper will address these findings in connection with the origin of the gamma-ray emission detected in IC 443.

Acknowledgements. We are very grateful to T. B. Humensky for kindly providing us the VERITAS significance contours, to Z. Zhang for the ${ }^{12} \mathrm{CO}(J=1-0)$ data, and to B.-C. Koo for the radio continuum image at $1420 \mathrm{MHz}$. We acknowledge the very useful comments of the anonymous referee. This publication makes use of data products from the Two Micron All Sky Survey, which is a joint project of the University of Massachusetts and the Infrared Processing and Analysis Center/California Institute of Technology, funded by the National Aeronautics and Space Administration and the National Science Foundation. The optical image used in this work is from the Second Palomar Observatory Sky Survey (POSS-II), which was made by the California Institute of Technology with funds from the National Science Foundation, the National Geographic Society, the Sloan Foundation, the Samuel Oschin Foundation, and the Eastman Kodak Corporation. This research has made use of the NASA's ADS Bibliographic Services. Data processing was carried out using the HOPE PC cluster at IAFE. This research was partially funded through CONICET (Argentina) grant PIP 112-200801-02166, ANPCYT-PICT (Argentina) grant 0902/07, and ANPCYT-PICT (Argentina) 08-0795 grant. Basic research in radio astronomy at the Naval Research Laboratory is supported by 6.1 base funds.

\section{References}

Abdo, A. A., Ackermann, M., Ajello, M., et al. 2010, ApJ, 712, 459

Acciari, V. A., Aliu, E., Arlen, T., et al. 2009, ApJ, 698, L133

Albert, J., Aliu, E., \& Anderhub, H. 2007, ApJ, 664, L87

Anderson, M. C., \& Rudnick, L. 1993, ApJ, 408, 514

Anderson, M. C., \& Rudnick, L. 1996, ApJ, 456, 234

Asaoka, I., \& Aschenbach, B. 1994, A\&A, 284, 573

Baars, J. W. M., Genzel, R., Pauliny-Toth, I. I. K., \& Witzel, A. 1977, A\&A, 61, 99

Baldwin, J. E., \& Dewhirst, D. W. 1954, Nature, 173, 164

Bell, A. R. 1978, MNRAS, 182, 147

Bennett, A. S. 1962, MmRAS, 68, 163

Bietenholz, M. F., Kassim, N., Frail, D. A., et al. 1997, ApJ, 490, 291

Blythe, J. H. 1957, MNRAS, 117, 652

Bocchino, F., \& Bykov, A. M. 2001, A\&A, 376, 248

Bondar, L. N., Krotikov, V. D., Stankevich, K. S., \& Tseitlin, N. M. 1965, Radiophys. Quant. Electron., 8, 437

Braude, S. Y., Lebedeva, O. M., Megn, A. V., Ryabov, B. P., \& Zhouck, I. N. 1969, MNRAS, 143, 289

Braun, R., \& Strom, R. G. 1986a, A\&AS, 63, 345

Braun, R., \& Strom, R. G. 1986b, A\&A, 164, 193

Bridle, A. H., \& Purton, C. R. 1968, AJ, 73, 717

Brogan, C. L., Lazio, T. J., Kassim, N. E., \& Dyer, K. K. 2005, AJ, 130, 148

Castelletti, G., Dubner, G., Golap, K., \& Goss, W. M. 2006, A\&A, 459, 535

Castelletti, G., Dubner, G., Brogan, C., \& Kassim, N. E. 2007, A\&A, 471, 537

Claussen, M. J., Frail, D. A., Goss, W. M., \& Gaume, R. A. 1997, ApJ, 489, 143

Colla, G., Fanti, C., Fanti, R., et al. 1971, AJ, 76, 953

Condon, J. J., Cotton, W. D., Greisen, E. W., et al. 1998, AJ, 115, 1693

Cornett, R. H., Chin, G., \& Knapp, G. R. 1977, A\&A, 54, 889

Cornwell, T. J., \& Perley, R. A. 1992, A\&A, 261, 353

Davies, M. M., Gelato-Volders, L., \& Westerhout, G. 1965,

Bull. Astron. Inst. Netherlands, 18, 42

Dickel, J. R. 1971, PASP, 83, 343

Dickel, J. R., \& McKinley, R. R. 1969, ApJ, 155, 67

Dickman, R. L., Snell, R. L., Ziurys, L. M., \& Huang, Y. 1992, ApJ, 400, 203

Dubner, G. M., Velázquez, P. F., Goss, W. M., \& Holdaway, M. A. 2000, AJ, 120,1933

Dulk, G. A., \& Slee, O. B. 1975, ApJ, 199, 61

Dwarakanath, K. S., Shevgaonkar, R. K., \& Sastry, C. V. 1982, J. Astrophys. Astron., 3, 207

Edge, D. O., Shakeshaft, J. R., McAdam, W. B., Baldwin, J. E., \& Archer, S. 1959, MmRAS, 68, 37

Erickson, W. C., \& Mahoney, M. J. 1985, ApJ, 290, 596

Esposito, J. A., Hunter, S. D., Kanbach, G., \& Sreekumar, P. 1996, ApJ, 461, 820

Fesen, R. A. 1984, ApJ, 281, 658

Fesen, R. A., \& Kirshner, R. P. 1980, ApJ, 242, 1023

Gaensler, B. M., Chatterjee, S., Slane, P. O., et al. 2006, ApJ, 648, 1037

Green, D. A. 1986, MNRAS, 221, 473

Guidice, D. A. 1969, Ph.D. Thesis, The Ohio State University

Hagen, J. P., Lilley, A. E., \& McClain, E. F. 1955, ApJ, 122, 361

Harris, D. E., \& Roberts, J. A. 1960, PASP, 72, 237 
Hewitt, J. W. 2009, Ph.D. Thesis, Northwestern University

Hewitt, J. W., Yusef-Zadeh, F., Wardle, M., Roberts, D. A., \& Kassim, N. E. 2006, ApJ, 652, 1288

Hill, L. E. 1972, MNRAS, 157, 419

Hirabayashi, H., \& Takahashi, T. 1972, PASJ, 24, 231

Hoffman, I. M., Goss, W. M., Brogan, C. L., Claussen, M. J., \& Richards, A. M. S. 2003, ApJ, 583, 272

Hogg, D. E. 1964, ApJ, 140, 992

Howard, III, W. E., \& Dickel, H. R. 1963, PASP, 75, 149

Humensky, T. B. 2008, in International Cosmic Ray Conference, 2, 835

Kassim, N. E. 1989, ApJ, 347, 915

Kassim, N. E., Perley, R. A., Dwarakanath, K. S., \& Erickson, W. C. 1995, ApJ, 455, L59

Kassim, N. E., Lazio, T. J. W., Erickson, W. C., et al. 2007, ApJS, 172, 686

Kawasaki, M. T., Ozaki, M., Nagase, F., et al. 2002, ApJ, 572, 897

Kellermann, K. I. 1964, ApJ, 140, 969

Kovalenko, A. V., Pynzar', A. V., \& Udal'Tsov, V. A. 1994, Astron. Rep., 38, 78

Kundu, M. R., \& Velusamy, T. 1968, MNRAS, 140, 173

Kundu, M. R., \& Velusamy, T. 1969, ApJ, 155, 807

Kundu, M. R., \& Velusamy, T. 1972, A\&A, 20, 237

Kuz'min, A. D., Levchenko, M. T., Noskova, R. I., \& Salomonovich, A. E. 1960, AZh, 37, 975

Lacey, C. K., Lazio, T. J. W., Kassim, N. E., et al. 2001, ApJ, 559, 954

Leahy, D. A. 2004, AJ, 127, 2277

Lee, J., Koo, B., Yun, M. S., et al. 2008, AJ, 135, 796
Lozinskaya, T. A. 1981, SvA Lett., 7, 17

Milne, D. K. 1971, Austr. J. Phys., 24, 429

Milne, D. K., \& Hill, E. R. 1969, Austr. J. Phys., 22, 211

Olbert, C. M., Clearfield, C. R., Williams, N. E., Keohane, J. W., \& Frail, D. A 2001, ApJ, 554, L205

Petre, R., Szymkowiak, A. E., Seward, F. D., \& Willingale, R. 1988, ApJ, 335, 215

Reach, W. T., \& Rho, J. 2000, ApJ, 544, 843

Reynolds, S. P., \& Ellison, D. C. 1992, ApJ, 399, L75

Rho, J., \& Petre, R. 1998, ApJ, 503, L167

Rho, J., Jarrett, T. H., Cutri, R. M., \& Reach, W. T. 2001, ApJ, 547, 885

Roger, R. S., Costain, C. H., \& Lacey, J. D. 1969, AJ, 74, 366

Roger, R. S., Costain, C. H., \& Stewart, D. I. 1986, A\&AS, 65, 485

Seeger, C. L., Westerhout, G., Conway, R. G., \& Hoekema, T. 1965, Bull. Astron. Inst. Netherlands, 18, 11

Shakeshaft, J. R., Ryle, M., Baldwin, J. E., Elsmore, B., \& Thomson, J. H. 1955 MmRAS, 67, 106

Steer, D. G., Dewdney, P. E., \& Ito, M. R. 1984, A\&A, 137, 159

Troja, E., Bocchino, F., \& Reale, F. 2006, ApJ, 649, 258

Viner, M. R., \& Erickson, W. C. 1975, AJ, 80, 931

Wang, Z. R., Asaoka, I., Hayakawa, S., \& Koyama, K. 1992, PASJ, 44, 303

Wanner, J. F. 1961, PASP, 73, 143

Westerhout, G. 1958, Bull. Astron. Inst. Netherlands, 14, 215

Williams, P. J. S., Kenderdine, S., \& Baldwin, J. E. 1966, MmRAS, 70, 53

Zhang, Z., Gao, Y., \& Wang, J. 2010, Science in China G: Phys. Astron., 53, 1357 